\title{
NUMERICAL APPROXIMATIONS OF THE RELATIVE REARRANGEMENT: THE PIECEWISE LINEAR CASE. APPLICATION TO SOME NONLOCAL PROBLEMS *,**
}

\author{
Jean-Michel Rakotoson $^{1}$ and Maria Luisa Seoane ${ }^{2}$
}

\begin{abstract}
We first prove an abstract result for a class of nonlocal problems using fixed point method. We apply this result to equations revelant from plasma physic problems. These equations contain terms like monotone or relative rearrangement of functions. So, we start the approximation study by using finite element to discretize this nonstandard quantities. We end the paper by giving a numerical resolution of a model containing those terms.
\end{abstract}

Mathematics Subject Classification. 76X05, 35R30, 35R35, 28A75, 46E30, 65L60, 65M60, 65N30.

Received: May 27, 1999. Revised: October 261999.

\section{INTRODUCTION}

In the mathematical models appearing in plasma physics either for the Tokamak models or the Stellerators models, the modelling equations may depend not only on the solution $u$ but also on the distribution function associated with that function, that is the volume of a level set $m_{u}(t)=$ meas $\{x \in \Omega, u(x)>t\}, t \in \mathbb{R},($ here, $\Omega$ is the mathematical domain). What is more, it might even depend on the generalized inverse of that function $m_{u}$, called the decreasing monotone rearrangement of $u$ and denoted by $u_{*}$ and its derivatives $u_{*}^{\prime}$ or $u_{*}^{\prime \prime}$. For instance, in the Tokamak models, Grad [25] and Shafranov [51], conjectured that the current flux $u$ (associated with the magnetic fields and the pressure) can satisfy an equation of the form

$$
-\Delta u(x)-\lambda u_{*}^{\prime \prime}\left(m_{u}(u(x))=f(x), x \in \Omega\right.
$$

( see also Temam [55,57]). More recently, in the case of a confined plasma in a Stellerator, Díaz-Rakotoson (see $[15,16]$ for the modelization, [19] for the mathematical justification and [5] for the numerical solution)

\footnotetext{
Keywords and phrases. Monotone decreasing and relative rearrangements, nonlocal problems, numerical approximations.

* Dedicated to Roger Temam for his 60th birthday

** This research has been partially supported by the University of Santiago.

1 Département de Mathématiques, Université de Poitiers, boulevard 3, Téléport 2, B.P. 179, 86960 Futuroscope, France.

2 Departamento de Matemática Aplicada, Facultad de Matemáticas, Campus Universitario, 15706 Santiago de Compostela, Spain.
} 
established that the current field $u$ satisfies the following equation:

$$
(\mathcal{P S})\left\{\begin{array}{l}
-\Delta u=a(x)\left[F_{v}^{2}-2 \int_{0}^{u_{+}(x)} p^{\prime}(t) b_{* u}\left(m_{u}(t)\right) \mathrm{d} t\right]_{+}^{1 / 2}+p^{\prime}(u)\left[b(x)-b_{* u}\left(m_{u}(u(x))\right)\right] \text { in } \Omega \\
u=\gamma \text { on } \partial \Omega
\end{array}\right.
$$

where

$$
b_{* u}=\lim _{t \searrow 0} \frac{(u+t b)_{*}-u_{*}}{t}
$$

is called, according to Mossino-Temam [34], the relative rearrangement of $b$ with respect to $u$ (see below for more details).

In this article, we wish to present a method for the numerical approximation of the monotone and relative rearrangements by using finite elements $\mathbb{P}_{1}$. Since very few results are known concerning the regularity of the derivatives of monotone and relative rearrangement functions, we shall only look at the convergence of the scheme according to what we know on the first derivative of the monotone rearrangement. (Almost nothing is known on the second derivatives of these quantities).

As an application in P.D.E, we choose a model which involves the first derivative of the monotone relative rearrangement and whose nonlinearities are of the same type as those in Grad-Shafranov in the Stellerator models, say:

$$
(\mathcal{P})\left\{\begin{array}{l}
-u^{\prime \prime}(x)-\lambda u_{*}^{\prime}\left(m_{u}(u(x))\right)-a(x)\left[F_{0}^{2}-\int_{m_{u}(0)}^{m_{u}\left(u_{+}(x)\right)} p^{\prime}\left(u_{*}\right) u_{*}^{\prime} b_{* u} \mathrm{~d} t\right]_{+}^{1 / 2}=f(x) \\
u(0)=u(1)=0
\end{array}\right.
$$

The variational problem associated with that problem reads:

$$
\left(\mathcal{P}_{v}\right)\left\{\begin{aligned}
& \text { Find } u \in H_{0}^{1}(0,1) \text { such that } \\
& \int_{0}^{1} u^{\prime}(x) v^{\prime}(x) \mathrm{d} x-\lambda \int_{0}^{1} u_{*}^{\prime}(s) v_{* u}(s) \mathrm{d} s-\int_{0}^{1} F(u)(x) v(x) \mathrm{d} x \\
&=\int_{0}^{1} f(x) v(x) \mathrm{d} x \quad \text { for all } v \in H_{0}^{1}(0,1)
\end{aligned}\right.
$$

where $F(u)(x)=a(x)\left[F_{0}^{2}-\int_{m_{u}(0)}^{m_{u}\left(u_{+}(x)\right)} p^{\prime}\left(u_{*}\right) u_{*}^{\prime} b_{* u} \mathrm{~d} t\right]_{+}^{1 / 2}, a$ and $b$ are in $\mathrm{L}^{\infty}(0,1), f \in \mathrm{L}^{2}(0,1), F_{0}>0$ is a constant and $p$ a $\mathrm{C}^{1}$-function with $p^{\prime}$ bounded for the sake of simplicity.

Along this paper, we use the fact that $u_{*} \in H^{1}(0,1)$ if $u \in H^{1}(0,1)$ and then $\frac{\mathrm{d}^{+} u_{*}}{\mathrm{~d} s}\left(m_{u}(u(x))\right)=u_{*}^{\prime}\left(m_{u}(u(x))\right)$ a.e.

In order to give a theoretical and numerical resolution of that problem, we shall consider a family of subspaces $V_{h}$ of $H_{0}^{1}(0,1)$ with finite dimension. Then, we shall prove the existence of a function $u_{h} \in V_{h}$ satisfying the following approximate problem

$$
\left(\mathcal{P}_{v}^{h}\right)\left\{\begin{aligned}
& \text { Find } u_{h} \in V_{h} \text { such that } \\
& \int_{0}^{1} u_{h}^{\prime}(x) v_{h}^{\prime}(x) \mathrm{d} x-\lambda \int_{0}^{1} u_{h *}^{\prime}(s)\left(v_{h}\right)_{* u_{h}}(s) \mathrm{d} s-\int_{0}^{1} F\left(u_{h}\right)(x) v_{h}(x) \mathrm{d} x \\
&=\int_{0}^{1} f(x) v_{h}(x) \mathrm{d} x \text { for all } v_{h} \in V_{h} .
\end{aligned}\right.
$$

In order to solve problem $\left(\mathcal{P}_{v}\right)$ and $\left(\mathcal{P}_{v}^{h}\right)$, we introduce an abstract result which will allow us to consider other nonlinearities than in $(\mathcal{P})$. 
This abstract result reads as follow:

Let $(V,\|\cdot\|)$ be a Hilbert space continuously and compactly imbedded in a Banach separable space $(H,|\cdot|)$. Assume that there exist a family of finite elements $V_{h} \subset V$ and a family of linear operators $\Pi_{h}: V \rightarrow V_{h}$ such that $\lim _{h \rightarrow 0}\left\|v-\Pi_{h} v\right\|=0$ for all $v \in V$. Consider $B: V \times V \rightarrow \mathbb{R}$ a bilinear coercive and continuous map and $G$ : $V \rightarrow H^{\prime}$ (dual of $H$ ) a nonlinear continuous map from $V$-strong into $H^{\prime}-*-\sigma\left(H^{\prime}, H\right)$ (weak-star topology), with $G$ having the following growth:

$$
|G(v)|_{*} \leq \lambda_{0}\|v\|+\lambda_{1} \quad 0<\lambda_{0}<\left(\inf _{\| v \mid=1} B[v, v]\right) \cdot\left(\inf _{|v|=1}\|v\|\right)
$$

Then:

i) There exists $u_{h} \in V_{h}$ such that

$$
B\left(u_{h}, v_{h}\right)=<G\left(u_{h}\right), v_{h}>, \quad \text { for all } v_{h} \in V_{h}
$$

ii) There exists $u \in V$ such that

$$
u_{h} \rightarrow u \text { in } V \text { strong } \quad B[u, v]=<G(u), v>\quad \text { for all } v \in V .
$$

It happens that for the kind of operators that we meet in the literature, the map $G$ is not continuous on the whole space $V$ but only on a subset $\mathcal{V}$ of $V$, containing all $V_{h}$; in which case we may assume that $H=H^{\prime}$ and then the continuity of $G$ can be restricted to $\mathcal{V} \subset V \rightarrow H$-weak, (for instance if $A \in \mathcal{L}\left(V, V^{\prime}\right)$ associated with the bilinear form $B$ then $\mathcal{V}=D(A)$ (domain of $A$ ) $\bigcup_{h} V_{h}$ induced by the norm of $V$ ). The above conclusion remains true provided that we show that $u \in \mathcal{V}$.

We shall apply statement i) and ii) with $V=H_{0}^{1}(0,1) H=\mathrm{L}^{2}(0,1)$

$$
B(u, v)=\int_{0}^{1} u^{\prime} v^{\prime}, \quad<G(u), v>=\lambda \int_{0}^{1} u_{*}^{\prime} v_{* u}+\int_{0}^{1} F(u)(x) v(x) \mathrm{d} x+\int_{0}^{1} f(x) v(x) \mathrm{d} x .
$$

The "main" difficulty will be to prove the continuity of $G$ from $V$-strong into $\mathrm{L}^{2}(0,1)$-weak. So we shall introduce some appropriate new lemmas (see Lem. 3.1 to Lem. 3.4).

As a consequence of this analysis, we derive a stability result for $u_{h}$ of the form:

$$
\left|u_{h}^{\prime}\right|_{\mathrm{L}^{2}(0,1)} \leq \frac{\sqrt{2}|f|_{\mathrm{L}^{2}}+|a|_{\infty} c}{1-|\lambda|} \text { for }|\lambda|<1 .
$$

We also obtain for statement ii) the convergence of the scheme.

Some qualitative properties for the solutions of the continuous and discrete problems are given: when $f$ is symmetric (that is $f(x)=f(1-x)$ ), we will show the existence of symmetric solutions. When $\lambda f<0, \lambda a<0$, then every solution $u$ of $\left(\mathcal{P}_{v}\right)$ is such that the set $\left\{x: u^{\prime}(x)=0\right\}$ is of measure zero. We shall also provide the rigidity matrix associated with the discretized problem.

For convenience for the reader, we start by recalling some useful notions on the monotone and relative rearrangements.

\section{Monotone And Relative REARRAngements of a FunCtion: DEFINITIONS AND PROPERTIES}

Since the numerical schemes that we shall present below are in one dimension, we restrict the introduction of this section to functions defined on $[0,1]$. Let $u$ be a real valued Lebesgue measurable function defined on 
] $0,1[$. For $t \in \mathbb{R}$, we denote by $\{u>t\}$ the set $\{x \in] 0,1[, u(x)>t\}$, and by $\{u=t\}$ the set $\{x \in] 0,1[, u(x)=t\}$. The Lebesgue measure of any measurable set $E$ is denoted $|E|$ or meas $(E)$; in particular, the measure of the above level sets are denoted by, $|u>t|,|u=t|$.

Definition 2.1. A measurable function $u$ on $] 0,1[$ has a plateau at a value $t$ if $|u=t|>0$.

We set $P(u)=\{x \in] 0,1[:|u=u(x)|>0\}$.

The distribution function associated with $u$ is the real valued function $t \in \mathbb{R} \rightarrow m_{u}(t)=|u>t|$.

Definition 2.2. For a measurable function $u$, the generalized inverse of its distribution function is called the monotone decreasing rearrangement, that is the function $u_{*}$ with finite value on on $] 0,1[$ given by: if $s \in[0,1[$, then $u_{*}(s)=\operatorname{Inf}\{t \in \mathbb{R}:|u>t| \leq s\}$ and $u_{*}(1)=\operatorname{essinf}\{u(x), x \in[0,1]\}$.

Properties of the monotone rearrangement

i) The monotone decreasing rearrangement $u_{*}$ of $u$ is equimeasurable to $u$, that is for all $t \in \mathbb{R}|u>t|=$ $\left|u_{*}>t\right|$. This implies in particular that the integral of $F(u)$ over the level set $\{u>t\}$ is equal to the integral of $F\left(u_{*}\right)$ over the level set $\left\{u_{*}>t\right\}$, whenever $F$ is a real valued Borel function with $F(u)$ integrable on $[0,1]$.

ii) If $u$ belongs to the Sobolev space $W^{1, p}(0,1), 1 \leq p \leq \infty$, then $u_{*}$ belongs to the same space and we have the inequality:

$$
\left|u_{*}^{\prime}\right|_{L^{p}(0,1)} \leq\left|u^{\prime}\right|_{L^{p}(0,1)},
$$

where we denote by $|\cdot|_{L^{p}(0,1)}$ the norm in the Lebesgue space $L^{p}(0,1)$.

For more details on these properties, see G. Talenti [52], Rakotoson-Temam [49], Mossino [36], Hardy Littlewood and Polya [27].

\subsection{Definition and properties of the relative rearrangement}

Let $u \in L^{1}(0,1)$ and $b \in L^{p}(0,1), 1 \leq p \leq \infty$. For a fixed $s$ in $[0,1]$, we denote by $B_{s}$ the restriction of $b$ to the level set $\left\{u=u_{*}(s)\right\}$. Define on $[0,1]$ the function $w$ by

$$
w(s)=\int_{\left\{u>u_{*}(s)\right\}} b(x) \mathrm{d} x+\int_{0}^{s-\left|u>u_{*}(s)\right|}\left(B_{s}\right)_{*}(t) \mathrm{d} t .
$$

Then, $w$ is in the Sobolev space $W^{1, p}(0,1)$ and the quotient $\frac{(u+t b)_{*}-u_{*}}{t}$ converges to $w^{\prime}$ as $t \searrow 0$, in $L^{p}(0,1)$-weak if $1<p<\infty$, in $L^{\infty}(0,1)$-weak-* if $p=\infty$ and for the topology $\sigma\left(L^{1}(0,1), L^{\infty}(0,1)\right)$ for $p=1$. The function $w^{\prime}$ is called the relative rearrangement of $b$ with respect to $u$ and is denoted by $b_{* u}$.

Properties of the relative rearrangement

Let $u \in L^{1}(0,1)$ and $b \in L^{p}(0,1), 1 \leq p \leq \infty$. Then,

i) The map $b \in L^{p}(0,1) \rightarrow b_{* u} \in L^{p}(0,1)$ is a contraction. In particular, we have the main inequality:

$$
\left|b_{* u}\right|_{L^{p}(0,1)} \leq|b|_{L^{p}(0,1)} .
$$

ii) If $\Phi$ is a nondecreasing function on $\mathbb{R}$, then $\Phi(u)_{* u}=\Phi\left(u_{*}\right)$ provided that $\Phi(u) \in L^{1}(0,1)$.

iii) If $u_{h}$ is a family of functions such that $u_{h}$ converges strongly to a function $u$ in $H^{1}(0,1)=W^{1,2}(0,1)$ and if $\left|\left\{x: u^{\prime}(x)=0\right\}\right|=\left|\left\{x: u_{h}^{\prime}(x)=0\right\}\right|=0$, then $b_{* u_{h}}$ converges strongly to $b_{* u}$ in $L^{p}(0,1)$, provided that $1 \leq p<\infty$.

One can also define the monotone and relative rearrangements associated with weighted functions. The definitions and properties given above can be carried naturally by making use of weighted spaces when necessary. In particular, if $a$ is a weight function then the distribution function $m_{u}^{a}$ of a Lebesgue measurable function $u$ 
with respect to the weight $a$ is $m_{u}^{a}(t)=\int_{\{u>t\}} a(x) \mathrm{d} x$. Its generalized inverse, that is the monotone decreasing rearrangement of $u$ with respect to $a$, is denoted $u_{*}^{a}$ and satisfies for $s \in\left[0, \int_{0}^{1} a(x) \mathrm{d} x[\right.$

$$
u_{*}^{a}(s)=\operatorname{Inf}\left\{t \in \mathbb{R}: m_{u}^{a}(t) \leq s\right\} .
$$

For more details on weighted relative rearrangement, we refer the reader to Rakotoson-Simon [48]. The link between relative rearrangement and weighted rearrangement is given in the following lemma:

Lemma 2.1. Let $u \in W^{1,1}(0,1)$ be such that $\left|\left\{x: u^{\prime}(x)=0\right\}\right|=0$ and let $b \in L^{\infty}(0,1)$ satisfy $\operatorname{essinf} b>0$. Then:

$$
b_{* u}\left(m_{u}(u(x))\right)=\frac{u_{*}^{\prime}\left(m_{u}(u(x))\right)}{\left(u_{*}^{b}\right)^{\prime}\left(m_{u}^{b}(u(x))\right)} .
$$

This lemma is proven in Díaz-Rakotoson [19].

We shall also use the following mean value formula; for a complete statement, we refer the interested reader to Mossino-Temam [34], Mossino [36], Rakotoson-Simon [47].

Lemma 2.2. Let $u, b$ be two functions in $L^{2}(0,1)$. Then, there exists a linear continuous operator from $L^{2}(0,1)$ into $L^{2}(0,1)$ denoted by $M_{u, b}$ such that for all $g \in L^{2}(0,1)$, one has:

$$
\int_{0}^{1} g(s) b_{* u}(s) \mathrm{d} s=\int_{0}^{1} M_{u, b}(g)(x) b(x) \mathrm{d} x .
$$

Furthermore, one has for $x \in] 0,1\left[\backslash P(u), M_{u, b}(g)(x)=g\left(m_{u}(u(x))\right)\right.$ : if $g$ vanishes on $P\left(u_{*}\right)$ then $M_{b, u}(g)(x)=$ 0 , for $x \in P(u)$.

\section{SOME THEORETICAL RESUlTS FOR SOME VARIATIONAL NONLOCAL PROBLEMS}

We begin this section by introducing an abstract result which will cover the resolution of $\left(\mathcal{P}_{v}\right)$ and of the discrete problem $\left(\mathcal{P}_{v}^{h}\right)$.

Through out this paper, we shall consider a Hilbert space $(V,\|\cdot\|)$ and a separable Banach space $(H,|\cdot|)$ satisfying:

H1) $V$ is a continuously and compactly imbedded in $H$; thus, $\inf _{|v|=1}\|v\|>0$.

H2) There exist a family of finite elements $V_{h} \subset V$ and a family of linear operators $\Pi_{h}$ such that $\lim _{h \rightarrow 0}\left\|v-\Pi_{h} v\right\|=$ 0 , for all $v \in V$.

We also consider a nonlinear map $G$ from $V$ into the dual space $H^{\prime}$ of $H$ satisfying:

H3) $G$ is continuous from $V$-strong into $H^{\prime}$-weak-star (i.e. for the topology $*-\sigma\left(H^{\prime}, H\right)$ ).

We then have the:

Theorem 3.1. Assume that H1) to H3) hold and let $B: V \times V \rightarrow \mathbb{R}$ be a bilinear form which is coercive (in the sense that $\alpha=\inf _{\|v\|=1} B(v, v)>0$ ), continuous (i.e. $\sup _{\|u\|=\|v\|=1} B(u, v)=M<+\infty$ ).

Assume that $G$ has the following growth:

H4) There exists $0<\lambda_{0}<\alpha \inf _{|v|=1}\|v\|$ and $0<\lambda_{1} \in \mathbb{R}$ such that: $|G(v)|_{*} \leq \lambda_{0}\|v\|+\lambda_{1}$ for all $v \in V$. Then,

i) there exists $u_{h} \in V_{h}$ such that

$$
B\left(u_{h}, v_{h}\right)=<G\left(u_{h}\right), v_{h}>, \quad \forall v_{h} \in V_{h}
$$

ii) there exist $u \in V$ and a (subsequence) $u_{h} \in V$ such that $u_{h}$ converges strongly to $u$ in $V$, where $u$ solves: $B(u, v)=<G(u), v>$ for all $v \in V$. 
Proof of Theorem 3.1

Let $m=\operatorname{dim} V_{h}$ and $\left\{\varphi_{1}, \cdots, \varphi_{m}\right\}$ be a basis of $V_{h}$. Define the following scalar product on $V_{h}$ for $v=\sum_{j=1}^{m} v_{j} \varphi_{j}$ and $w=\sum_{j=1}^{m} w_{j} \varphi_{j}: \quad[v, w]=\sum_{j=1}^{m} v_{j} w_{j}$. We introduce the map $T_{m}: V_{h} \rightarrow V_{h}$ by setting $T_{m} v=\sum_{j=1}^{m}\left[B\left(v, \varphi_{j}\right)-<G(v), \varphi_{j}>\right] \varphi_{j}$. To prove statement i) of Theorem 3.1, we see that for all $v \in V_{h}$

$$
\begin{aligned}
{\left[T_{m} v, v\right]=B(v, v)-\langle G(v), v>} & \geq \alpha\|v\|^{2}-\lambda_{0}\|v\| \cdot|v|-\lambda_{1}|v| \\
& \geq\left(\alpha \inf _{|z|=1}\|z\|-\lambda_{0}\right)\|v\||v|-\lambda_{1}|v|
\end{aligned}
$$

Thus, $\left[T_{m} v, v\right] \rightarrow+\infty$ as $[v, v] \rightarrow+\infty$. Furthermore, $T_{m}$ is continuous, the continuity of $B$ and $G$ yield that. We conclude with Brouwer's fixed point theorem to obtain: the existence of $u_{h} \in V_{h}$ such that $T_{m} u_{h}=$ $0, B\left(u_{h}, v_{h}\right)=<G\left(u_{h}\right), v_{h}>$ for all $v_{h} \in V_{h}$ and $\left\|u_{h}\right\| \leq$ Constant $=\frac{\lambda_{1}}{\alpha \inf _{|z|=1}\|z\|-\lambda_{0}}$.

We consider $u \in V$ and a subsequence still denoted by $u_{h}$ such that $u_{h} \rightarrow u$ weakly in $V$ and $H$-strong and $G\left(u_{h}\right) \rightarrow \ell_{u}$ in $H^{\prime}$-weak-star.

Let $v \in V$. Then, one has:

$$
\begin{gathered}
B\left(u_{h}, \Pi_{h} v\right)=<G\left(u_{h}\right), \Pi_{h} v> \\
\left|B\left(u_{h}, \Pi_{h} v\right)-B\left(u_{h}, v\right)\right| \leq(\text { Constant }) \cdot\left\|v-\Pi_{h} v\right\| \\
\left|<G\left(u_{h}\right), \Pi_{h} v>-<G\left(u_{h}\right), v>\right| \leq C_{0}\left\|v-\Pi_{h} v\right\| .
\end{gathered}
$$

From relation (1) to (3), we deduce:

$$
B(u, v)=\lim _{h \rightarrow 0} B\left(u_{h}, \Pi_{h} v\right)=\lim _{h \rightarrow 0}<G\left(u_{h}\right), v>=<\ell_{u}, v>
$$

Let us show that $\lim _{h \rightarrow 0}\left\|u_{h}-u\right\|=0$. It suffices to show that $\lim _{h \rightarrow 0} B\left(u_{h}, u_{h}\right)=B(u, u)$. One has:

$$
\lim _{h \rightarrow 0}<G\left(u_{h}\right), u_{h}>=<\ell_{u}, u>\text { since }\left|u_{h}-u\right| \underset{h \rightarrow 0}{\longrightarrow} 0,
$$

that is $\lim _{h \rightarrow 0} B\left(u_{h}, u_{h}\right)=B(u, u)$. By the continuity of $G$, we deduce that $\ell_{u}=G(u)$.

Remark 3.1. Suppose that the map $G$ is only continuous on a subset $\mathcal{V}$ of $V$ containing all the $V_{h}$. In that case, statement i) as well as the strong convergence remain true. Furthermore, if we can show that $u \in \mathcal{V}$, then the conclusion of the second statement ii) is also true. Let us give an example of such a situation:

We replace assumption H3) by the following one:

H5) Let $A$ be the linear continuous operator from $V$ to $V^{\prime}$ defined by $\langle A v, w\rangle=B(v, w)$ for all $v, w$ and let us denote by $D(A)$ its domain. We assume that $H=H^{\prime}, G$ maps $V$ into $H$ and its restriction to $D(A) \cup\left(\bigcup_{h>0} V_{h}\right)=\mathcal{V}$ is continuous from $(\mathcal{V},\|\cdot\|)$-strong into $H$-weak. 
Then:

Theorem 3.2. Assume that H1), H2), H4), and H5) hold. If B is the same bilinear form as in Theorem 3.1, then we have the same conclusions as in Theorem 3.1.

Proof. The proof of statement i) is the same as in Theorem 3.1, while for the statement ii), the function $u$ is found as a strong limit of a sequence $u_{h}$, and belongs to $D(A)$ since $A u=\ell_{u} \in H$. Thus, $G\left(u_{h}\right) \rightarrow G(u)$ in $H$-weak, which implies that $\ell_{u}=G(u)$.

Other situations will be given in the applications below when $\mathcal{V}$ is not a vector space. In order to verify the hypotheses satisfied by $G$ when it involves the relative rearrangement $b_{* u}$, we introduce the following weakconvergence:

Lemma 3.1. Let $v \in \mathrm{L}^{1}(0,1), \quad \theta=\chi_{\mathrm{P}\left(\mathrm{v}_{*}\right)}$ the characteristic function of $P\left(v_{*}\right)$. If $v_{n}$ tends to $v$ in $\mathrm{L}^{1}(0,1)$ strong then the sequence $(1-\theta) b_{* v_{n}}$ converges weakly to $(1-\theta) b_{* v}$ in $L^{p}$-weak whenever $1<p<+\infty$ and $b \in \mathrm{L}^{\mathrm{p}}(0,1)$.

Proof. Let $\varphi \in \mathcal{C}[0,1]$. Without loss of generality, we may assume that $\theta$ is continuous and equal to 1 on $P\left(v_{*}\right)$. Then, by the mean value theorem, we have

$$
\int_{0}^{1} \varphi(1-\theta) b_{* v_{n}} \mathrm{~d} s=\int_{0}^{1} M_{v_{n}, b}(\varphi(1-\theta)) b(x) \mathrm{d} x .
$$

For convenience, we introduce the following notations, for $x \in] 0,1[$ :

$$
\begin{array}{ll}
\beta_{n}(x)=\left|v_{n}>v_{n}(x)\right| & \gamma_{n}(x)=\beta_{n}(x)+\left|v_{n}=v_{n}(x)\right| \\
\beta(x)=|v>v(x)| & \gamma(x)=\beta(x)+|v=v(x)| .
\end{array}
$$

From the definition of the mean value operator, one can deduce that:

$$
\inf _{\sigma \in\left[\beta_{n}(x), \gamma_{n}(x)\right]} \varphi(1-\theta)(\sigma) \leq M_{v_{n}, b}(\varphi(1-\theta))(x) \leq \sup _{\sigma \in\left[\beta_{n}(x), \gamma_{n}(x)\right]} \varphi(1-\theta)(\sigma) .
$$

From relation $(6)$, we then deduce for all $x$ :

$$
\begin{aligned}
& \inf _{\sigma \in[\beta(x), \gamma(x)]} \varphi(1-\theta)(\sigma) \leq \liminf _{n} M_{v_{n}, b}(\varphi(1-\theta))(x) \leq \\
& \limsup _{n} M_{v_{n}, b}(\varphi(1-\theta))(x) \leq \sup _{\sigma \in[\beta(x), \gamma(x)]} \varphi(1-\theta)(\sigma) .
\end{aligned}
$$

If $x \in P(v)$, then $[\beta(x), \gamma(x)] \subset P\left(v_{*}\right)$ and $(1-\theta)(\sigma)=0$ for $\sigma \in[\beta(x), \gamma(x)]$, which implies that $\lim _{n \rightarrow \infty} M_{v_{n}, b}(\varphi(1-\theta))(x)=0$. If $x \notin P(v)$, then $\beta(x)=\gamma(x) \notin P\left(v_{*}\right)$ and then $\lim _{n \rightarrow \infty} M_{v_{n}, b}(\varphi(1-\theta))(x)=$ $\varphi(\beta)(x)$. We have shown that for all $x \in] 0,1[$,

$$
\lim _{n \rightarrow \infty} M_{v_{n}, b}(\varphi(1-\theta))(x)=(\varphi(1-\theta))(\beta(x))=M_{v, b}(\varphi(1-\theta))(x) .
$$

We conclude with Lebesgue's and the mean value theorems to find:

$$
\lim _{n \rightarrow \infty} \int_{0}^{1} \varphi(1-\theta) b_{* v_{n}} \mathrm{~d} s=\int_{0}^{1} M_{v, b}(\varphi(1-\theta)) b(x) d x=\int_{0}^{1} \varphi(1-\theta) b_{* v} \mathrm{~d} s
$$

We end by a classical density argument. 
Remark 3.2. This lemma is true in any dimension and also for $p=+\infty$.

Lemma 3.2. Let $v \in \mathrm{L}^{1}(0,1), \theta=\chi_{P\left(v_{*}\right)}$ be the characteristic function of $P\left(v_{*}\right)$ and $v_{n}$ be a sequence of $L^{1}(0,1)$ converging to $v$, almost everywhere and in $L^{1}(0,1)$. We set, for $x \in[0,1]$

$$
\left.I\left(v_{n}\right)(x)=\left[m_{v_{n}}\left(v_{n+}(x)\right)\right), m_{v_{n}}(0)\right] \quad(\operatorname{resp} I(v)(x))
$$

and we denote by $\chi_{I\left(v_{n}\right)(x)}\left(\right.$ resp $\left.\chi_{I(v)(x)}\right)$ the characteristic function of $I\left(v_{n}\right)(x)($ resp $I(v)(x))$. Then, for all $\sigma \in[0,1], \sigma \neq\left|v>v_{+}(x)\right|, \sigma \neq|v>0|$, one has

$$
\lim _{n \rightarrow+\infty}(1-\theta)(\sigma) \chi_{I\left(v_{n}\right)(x)}(\sigma)=(1-\theta)(\sigma) \chi_{I(v)(x)}(\sigma) .
$$

Proof. Let $\sigma \in[0,1], \sigma \neq|v>0|$ and $\sigma \neq\left|v>v_{+}(x)\right|$.

Note that we always have

$$
|v>0| \leq \lim \inf \left|v_{n}>0\right| \leq \lim \sup \left|v_{n}>0\right| \leq|v \geq 0|
$$

and,

$$
\left|v>v_{+}(x)\right| \leq \lim \inf \left|v_{n}>v_{n_{+}}(x)\right| \leq \lim \sup \left|v_{n}>v_{n_{+}}(x)\right| \leq\left|v \geq v_{+}(x)\right|
$$

So, if $\sigma<|v>0|$ or $\sigma>\left|v \geq v_{+}(x)\right|$, then for large $n, \chi_{I\left(v_{n}\right)(x)}(\sigma)=\chi_{I(v)(x)}(\sigma)=0$.

If $\sigma \in]|v \geq 0|,\left|v>v_{+}(x)\right|\left[\right.$, then the same conclusion holds, that is $\chi_{I\left(v_{n}\right)(x)}(\sigma)=\chi_{I(v)(x)}(\sigma)=1$ for large $n$.

If $\sigma \in]|v>0|,|v \geq 0|]$ and $|v=0|>0$, or $\left.\sigma \in]\left|v>v_{+}(x)\right|,\left|v \geq v_{+}(x)\right|\right]$ and $\left|v=v_{+}(x)\right|>0$, then $(1-\theta)(\sigma)=0$.

Lemma 3.3. Under the same assumptions as in Lemma 3.2, if $v_{h} \in \mathrm{H}_{0}^{1}(0,1)$ converges strongly to $v$, then for all $x \in[0,1]$

$$
\lim _{h \rightarrow 0} \int_{0}^{1}(1-\theta) \chi_{I\left(v_{h}\right)(x)}(\sigma) v_{h_{*}}^{\prime}(\sigma) p^{\prime}\left(v_{h_{*}}\right)(\sigma) b_{* v_{h}}(\sigma)=\int_{0}^{1}(1-\theta) \chi_{I(v)(x)}(\sigma) v_{*}^{\prime}(\sigma) p^{\prime}\left(v_{*}\right)(\sigma) b_{* v}(\sigma) \mathrm{d} \sigma
$$

whenever $b \in \mathrm{L}^{2}(0,1), p^{\prime} \in C(\mathbb{R})$ and $\left|p^{\prime}(t)\right| \leq c_{2}$, for all $t \in \mathbb{R}, p(0)=0$.

Proof. From Coron's result (see [13]), we have $v_{h_{*}}^{\prime} \rightarrow v_{*}^{\prime}$ in $\mathrm{L}^{2}(0,1)$ and $p^{\prime}\left(v_{h_{*}}\right) \rightarrow p^{\prime}\left(v_{*}\right)$ in $\mathrm{L}^{2}(0,1)$.

Then, from the above result, we have $(1-\theta) \chi_{I\left(v_{h}\right)(x)}(\cdot) v_{h_{*}}^{\prime} p^{\prime}\left(v_{h_{*}}\right) \rightarrow(1-\theta) \chi_{I(v)(x)}(\cdot) v_{*}^{\prime} p^{\prime}\left(v_{*}\right)$ in $\mathrm{L}^{2}(0,1)$-strong and $(1-\theta) b_{* v_{h}} \rightarrow(1-\theta) b_{* v}$ weakly in $\mathrm{L}^{2}(0,1)$.

Since $(1-\theta)^{2}=(1-\theta)$ (remember that $\theta$ is a characteristic function), we then deduce the result from the two last convergences.

As a consequence of this lemma, we have the,

Corollary 3.1 (of Lemma 3.3). Under the same assumptions as in Lemma 3.3, the map $v \in H^{1}(0,1) \rightarrow F(v) \in$ $\mathrm{L}^{2}(0,1)$ is continuous for the strong topology. Here, $F(v)(x)=a(x)\left[F_{0}^{2}-\int_{m_{v}(0)}^{m_{v}\left(v_{+}(x)\right)} p^{\prime}\left(v_{*}\right) v_{*}^{\prime} b_{* v} \mathrm{~d} t\right]_{+}^{1 / 2}$.

Proof. Let $v_{n}$ be a sequence converging to a function $v$ in $H^{1}(0,1)$. Let $\theta($.$) be the characteristic function of$ $P\left(v_{*}\right)$. Since $v_{*}^{\prime}(\sigma)=0$ whenever $\sigma \in P\left(v_{*}\right)$, one then has using Coron's continuity result that

$$
\lim _{n \rightarrow \infty} \int_{0}^{1}\left|v_{n *}^{\prime}(s) \theta(s)\right|^{2} \mathrm{~d} s=0 .
$$


Using the fact that

$$
\left|b_{* v_{n}}\right|_{L^{2}} \leq|b|_{L^{2}}
$$

we derive that:

$$
\lim _{n \rightarrow \infty} \int_{0}^{1} \theta(s) v_{n *}^{\prime}(s) p^{\prime}\left(v_{n *}(s) \chi_{I\left(v_{n}\right)(x)}(s) b_{* v_{n}} \mathrm{~d} s=0\right.
$$

Thus, if we write

$$
\begin{aligned}
\int_{m_{v_{n}}(0)}^{m_{v_{n}}\left(v_{n+}(x)\right)} p^{\prime}\left(v_{n *}\right) v_{n *}^{\prime} b_{* v_{n}} \mathrm{~d} t= & \int_{0}^{1}(1-\theta(s)) v_{n *}^{\prime}(s) p^{\prime}\left(v_{n *}(s) \chi_{I\left(v_{n}\right)(x)}(s) b_{* v_{n}}(s) \mathrm{d} s\right. \\
& +\int_{0}^{1} \theta(s) v_{n *}^{\prime}(s) p^{\prime}\left(v_{n *}(s) \chi_{I\left(v_{n}\right)(x)}(s) b_{* v_{n}}(s) \mathrm{d} s\right.
\end{aligned}
$$

we can apply Lemma 3.3 and the above convergence to find that $F\left(v_{n}\right)(x) \rightarrow F(v)(x) \forall x$. From the main estimates on the monotone rearrangement and relative rearrangement (see Sect. 1), we derive that:

$$
\left|F\left(v_{n}\right)(x)\right| \leq|a|_{\infty}\left[F_{0}+|b|_{L^{2}(0,1)}^{1 / 2}\left|p\left(v_{n}\right)\right|_{H^{1}(0,1)}^{1 / 2}\right] \leq \text { constant }
$$

We conclude with Lebesgue's theorem.

Lemma 3.4. $\quad$ i) For any $v \in H_{0}^{1}(0,1)$, the map $b \in L^{2}(0,1) \rightarrow \int_{0}^{1} v_{*}^{\prime}(s) b_{* v} \mathrm{~d} s$ is linear and continuous.

ii) For a fixed $b \in L^{2}(0,1)$, the map $v \in W^{1,2}(0,1) \rightarrow \int_{0}^{1} v_{*}^{\prime}(s) b_{* v} \mathrm{~d} s$ is continuous for the strong topology of $W^{1,2}(0,1)$.

Proof. From the mean value theorem (see Lem. 2.2), one has $M_{v, b}\left(v_{*}^{\prime}\right)(x)=v_{*}^{\prime}\left(m_{v}(v(x))\right)$. Thus

$$
\int_{0}^{1} v_{*}^{\prime}(s) b_{* v} \mathrm{~d} s=\int_{0}^{1} v_{*}^{\prime}\left(m_{v}(v(x))\right) b(x) \mathrm{d} x
$$

which shows the linearity of the map. The continuity is a consequence of Schwartz's inequality and the main inequalities for $v_{*}^{\prime}$ and $b_{* v}$ (see Sect. 1, properties of the monotone and relative rearrangements). For the statement ii), the proof is similar to that performed in Corollary 3.1 of Lemma 3.3.

Theorem 3.3. Let $V_{h}$ be a family of finite elements in $H_{0}^{1}(0,1)$ such that there exists a family of linear operators $\Pi_{h}$ from $H_{0}^{1}(0,1)$ in $V_{h}$ satisfying $\lim _{h \rightarrow 0}\left|v-\Pi_{h} v\right|_{H_{0}^{1}(0,1)}=0$. Then, there exist a solution $u_{h}$ of $\left(\mathcal{P}_{v}^{h}\right)$ and a solution $u \in H_{0}^{1}(0,1) \cap H^{2}(0,1)$ of $\left(\mathcal{P}_{v}\right)$, provided that $|\lambda|<1$.

Proof. We define a function $G: H_{0}^{1}(0,1) \rightarrow L^{2}(0,1)$ by setting

$$
(G(v), \varphi)=\lambda \int_{0}^{1} v_{*}^{\prime}(s) \varphi_{* v}(s) \mathrm{d} s+\int_{0}^{1} F(v)(x) \varphi(x) \mathrm{d} x+\int_{0}^{1} f(x) \varphi(x) \mathrm{d} x
$$

for all $v \in H_{0}^{1}(0,1)$ for all, $\varphi \in L^{2}(0,1)$. We have

$$
|G(v)|_{L^{2}(0,1)} \leq|\lambda|\left|v^{\prime}\right|_{L^{2}(0,1)}+|a|_{\infty}\left[F_{0}+|b|_{L^{2}(0,1)}^{1 / 2}|p(v)|_{H_{0}^{1}(0,1)}^{1 / 2}\right]+|f|_{L^{2}(0,1)} .
$$


By Young's inequality, one deduces that: $\forall \varepsilon>0, \exists c_{\varepsilon}$ such that:

$$
|G(v)|_{L^{2}(0,1)} \leq(|\lambda|+\varepsilon)|v|_{H_{0}^{1}(0,1)}+|a|_{\infty} c_{\varepsilon} .
$$

Furthermore, the map $G$ is continuous from $H_{0}^{1}(0,1)$-strong into $L^{2}(0,1)$-weak. (This is a consequence of Corollary 3.1 of Lem. 3.3 and Lem. 3.4). Setting $B(v, \varphi)=\int_{0}^{1} v^{\prime} \varphi^{\prime}, V=H_{0}^{1}(0,1), H=L^{2}(0,1)$, we then have, choosing $0<\epsilon<1-|\lambda|$ :

$$
|\lambda|+\varepsilon<1 \leq \inf _{|v|_{V}=1} B(v, v) \cdot \inf _{|v|_{H}=1}|v|_{V} .
$$

We can apply Theorem 3.1 to deduce that there exists $u_{h} \in V_{h}$ such that $B\left(u_{h}, v_{h}\right)=\left(G\left(u_{h}\right), v_{h}\right) \forall v_{h} \in V_{h}$ and $u \in V$ such that $B(u, v)=(G(u), v) \forall v \in V$. Since $-u "=G(u) \in L^{2}(0,1)$, we deduce that $u \in H^{2}(0,1)$.

From Theorem 3.1, we also deduce a stability result for the discrete problem, that can be written as:

$$
\left|u_{h}^{\prime}\right|_{\mathrm{L}^{2}(0,1)} \leq \frac{\sqrt{2}|f|_{\mathrm{L}^{2}}+|a|_{\infty} c}{1-|\lambda|} \text { for }|\lambda|<1,
$$

where $c$ depends only on $a, b, F_{0}$.

As an application of Theorem 3.2, one has the following existence result.

Theorem 3.4. Let $f \in L^{2}(0,1), b \in L^{\infty}(0,1) g \in C(\mathbb{R}), g \geq 0, b \geq 0$ and $f<0$.

Then, there exists $u \in H^{2}(0,1) \cap H_{0}^{1}(0,1)$ (non trivial) solution of

$$
\int_{0}^{1} u^{\prime} \varphi^{\prime}+\int_{0}^{1} g\left(u_{*}\right) b_{* u} \varphi_{* u}=\int_{0}^{1} f \varphi
$$

for all $\varphi \in H_{0}^{1}(0,1)$.

Proof. We set $\varphi_{j}(x)=\sqrt{2} \sin (j \pi x), H=L^{2}(0,1), V=H_{0}^{1}(0,1)$, and $V_{m}=\operatorname{span}\left\{\varphi_{1}, \varphi_{2}, \cdots, \varphi_{m}\right\}$

$$
B(v, \varphi)=\int_{0}^{1} v^{\prime} \varphi^{\prime}=<A v, \varphi>
$$

with $D(A)=H_{0}^{1}(0,1) \bigcap H^{2}(0,1), \mathcal{V}=\left\{v \in D(A)\right.$, measure $\left.\left\{x: v^{\prime}(x)=0\right\}=0\right\} \bigcup\{0\}$. For $v \in H_{0}^{1}(0,1)$, we define $G(v)$ as a solution of $(G(v), \varphi)=-\int_{0}^{1} g\left(v_{*}\right) b_{* v}(s) \varphi_{* v}(s) \mathrm{d} s+\int_{0}^{1} f \varphi$ for all $\varphi \in L^{2}(0,1)$. Therefore, $G(v) \in L^{2}(0,1)$ and one has

$$
|G(v)|_{L^{2}(0,1)} \leq|g(v)|_{\infty}|b|_{\infty}+|f|_{L^{2}(0,1)}
$$

By Theorem 1 of [46] and Lemma 3.1, we infer $G$ is continuous from $\left(\mathcal{V},|\cdot|_{H_{0}^{1}(0,1)}\right)$ into $L^{2}(0,1)$-weak. Thus H4) is satisfied. Assumptions H1), to H2) are easily checked. Since $b \geq 0, g \geq 0$, then there exists $h \geq 0$ such that $-u^{\prime \prime}(x)+h(x)=f(x)$, and if $\left|u^{\prime}=0\right|>0$, then $h(x)=f(x)$ a.e. on the set $\left\{u^{\prime}=0\right\}$, which contradicts the fact that $f<0$. Therefore, $u \in \mathcal{V}$. We may then apply Remark 1 of Theorem 3.1. Arguing by contradiction, we see that $u \neq 0$.

\section{Some qualitative aspects of a solution of $\left(\mathcal{P}_{v}\right)$ And $\left(\mathcal{P}_{v}^{h}\right)$}

We start this section by studying the existence of symmetric solutions (with respect to $\frac{1}{2}$ ) whenever $f$ is symmetric. 
For a measurable function $v$ on $[0,1]$, we set $v^{s}(x)=v(1-x), x \in[0,1]$.

We shall say that a function $v \in L^{1}(0,1)$ is symmetric if $v(x)=v(1-x)$ a.e. We set

$$
\begin{aligned}
L_{s}^{2}(0,1)= & \left\{v \in L^{2}(0,1) \text { such that } v \text { is symmetric }\right\} \\
& H_{0, s}^{1}(0,1)=H_{0}^{1}(0,1) \cap L_{s}^{2}(0,1) .
\end{aligned}
$$

Proposition 4.1. Let $u$ be a solution of $(\mathcal{P})$. Then, the function $u^{s}$ is also a solution whenever $f \in L_{s}^{2}(0,1)$, $a$ and $b$ are also symmetric.

Proof. First, we observe that $u$ and $u^{s}$ are equimeasurable (i.e. $m_{u}=m_{u^{s}}$ ). Thus, $u_{*}^{s}=u_{*}$ and for all $v \in L^{2}(0,1)$, we have from the mean value theorem and a change of variables,

$$
\begin{aligned}
\int_{0}^{1} u_{*}^{\prime}(t) v_{* u}^{s}(t) d t & =\int_{0}^{1}\left(u_{*}^{s}\right)^{\prime}\left(\left|u^{s}>u(x)\right|\right) v^{s}(x) \mathrm{d} x \\
& =\int_{0}^{1}\left(u_{*}^{s}\right)^{\prime}\left(\left|u^{s}>u^{s}(x)\right|\right) v(x) \mathrm{d} x \\
& =\int_{0}^{1}\left(u_{*}^{s}\right)^{\prime}(t) v_{* u^{s}}(t) \mathrm{d} t .
\end{aligned}
$$

If $b$ is symmetric, then $b_{* u^{s}}^{s}=b_{* u^{s}}=b_{* u}$. By a simple change of variables, we then have:

$$
\int_{0}^{1} F(u)(x) v^{s}(x) \mathrm{d} x=\int_{0}^{1} F\left(u^{s}\right)(x) v(x) \mathrm{d} x .
$$

For any $v \in H_{0}^{1}(0,1)$, we have $v^{s} \in H_{0}^{1}(0,1)$ and

$$
\int_{0}^{1} u^{\prime}(x)\left(v^{s}\right)^{\prime}(x) \mathrm{d} x-\lambda \int_{0}^{1} u_{*}^{\prime}(t) v_{* u}^{s}(t) \mathrm{d} t-\int_{0}^{1} F(u)(x) v^{s}(x) \mathrm{d} x=\int_{0}^{1} f(x) v^{s}(x) \mathrm{d} x,
$$

that is

$$
-\int_{0}^{1} u^{\prime}(x) v^{\prime}(1-x) \mathrm{d} x-\lambda \int_{0}^{1}\left(u_{*}^{s}\right)^{\prime}(t) v_{* u^{s}}(t) \mathrm{d} t-\int_{0}^{1} F\left(u^{s}\right)(x) v(x) \mathrm{d} x=\int_{0}^{1} f^{s}(x) v(x) \mathrm{d} x .
$$

So if $f^{s}=f$, then this last equation reads

$$
\int_{0}^{1}\left(u^{s}\right)^{\prime}(x) v^{\prime}(x) \mathrm{d} x-\lambda \int_{0}^{1}\left(u_{*}^{s}\right)^{\prime}(t) v_{* u^{s}}(t) \mathrm{d} t-\int_{0}^{1} F\left(u^{s}\right)(x) v(x) \mathrm{d} x=\int_{0}^{1} f(x) v(x) \mathrm{d} x,
$$

that is $u^{s}$ is a solution of $(\mathcal{P})$.

Theorem 4.1 (Existence of a symmetric solution). If $f \in L_{s}^{2}(0,1)$ and $a$ and $b$ are also symmetric, then there exists a symmetric solution $u \in H_{0}^{1}(0,1) \cap H^{2}(0,1)$.

Proof. The set $H_{0, s}^{1}(0,1)$ of symmetric functions is a closed subset of $H_{0}^{1}(0,1)$. Thus, this space is a Hilbert separable space endowed with the usual scalar product of $H_{0}^{1}(0,1)$. So, let $\left\{\varphi_{1}, \cdots, \varphi_{m}, \cdots\right\}$ be a hilbertian basis of $H_{0, s}^{1}(0,1)$. We define $V_{m}^{s}$ to be the vector space spanned by $\left\{\varphi_{1}, \cdots, \varphi_{m}\right\}$. Reconsidering the same operator $T_{m}$ as in Theorem 2.1 defined by:

$$
T_{m} v(x)=\sum_{j=1}^{m} a_{j} \varphi_{j}(x)
$$


with

$$
a_{j}=\int_{0}^{1} v^{\prime}(x) \varphi_{j}^{\prime}(x) \mathrm{d} x-\lambda \int_{0}^{1} v_{*}^{\prime}(x) \varphi_{j * v}(x) \mathrm{d} x-\int_{0}^{1} F(v)(x) \varphi_{j}(x) \mathrm{d} x-\int_{0}^{1} f(x) \varphi_{j}(x) \mathrm{d} x .
$$

We have, since $\varphi_{j}$ is symmetric, $T_{m} v \in V_{m}^{s}$. So, the same argument as in the preceding paragraph (see Theor. 3.1 using Brouwer's fixed point theorem) shows the existence of $u_{m}^{s} \in V_{m}^{s}$ such that $T_{m} u_{m}^{s}=0$. Thus, there exist a function $u \in H_{0, s}^{1}(0,1)$ and a subsequence still denoted by $u_{m}^{s}$ such that $u_{m}^{s} \rightarrow u$ in $H_{0, s}^{1}(0,1)$ weak and uniformly in $C[0,1]$. The function $u$ solves for all $v \in H_{0, s}^{1}(0,1)$

$$
\int_{0}^{1} u^{\prime}(x) v^{\prime}(x) \mathrm{d} x-\lambda \int_{0}^{1} u_{*}^{\prime}(t) v_{* u}(t) \mathrm{d} t-\int_{0}^{1} F(u)(x) v(x) \mathrm{d} x=\int_{0}^{1} f(x) v(x) \mathrm{d} x .
$$

Let $v \in H_{0}^{1}(0,1)$. Then, $w=\frac{v+v^{s}}{2} \in H_{0, s}^{1}(0,1)$. So, one has from relation (17):

$$
\begin{aligned}
& \frac{1}{2}\left[\int_{0}^{1} u^{\prime}(x) v^{\prime}(x) \mathrm{d} x-\lambda \int_{0}^{1} u_{*}^{\prime}(t) v_{* u}(t) \mathrm{d} t-\int_{0}^{1} F(u)(x) v(x) \mathrm{d} x-\int_{0}^{1} f v\right] \\
& +\frac{1}{2}\left[\int_{0}^{1} u^{\prime}(t)\left(v^{s}\right)^{\prime}(t) \mathrm{d} t-\lambda \int_{0}^{1} u_{*}^{\prime} v_{* u}^{s}-\int_{0}^{1} F\left(u^{s}\right)(x) v(x) \mathrm{d} x-\int_{0}^{1} f v^{s}\right]=0 .
\end{aligned}
$$

By a change of variables, one has:

$$
\begin{aligned}
& \int_{0}^{1} u^{\prime}(x)\left(v^{s}\right)^{\prime}(x) \mathrm{d} x=\int_{0}^{1}\left(u^{s}\right)^{\prime}(x) v^{\prime}(x) \mathrm{d} x=\int_{0}^{1} u^{\prime}(x) v^{\prime}(x) \mathrm{d} x \\
& \int_{0}^{1} f(x) v^{s}(x) \mathrm{d} x=\int_{0}^{1} f^{s}(x) v(x) \mathrm{d} x=\int_{0}^{1} f(x) v(x) \mathrm{d} x \\
& \int_{0}^{1} F\left(u^{s}\right)(x) v^{s}(x) \mathrm{d} x=\int_{0}^{1} F(u)(x) v^{s}(x) \mathrm{d} x=\int_{0}^{1} F\left(u^{s}\right)(x) v(x) \mathrm{d} x=\int_{0}^{1} F(u)(x) v(x) \mathrm{d} x .
\end{aligned}
$$

So by relations $(13,18,19)$, one finally obtains:

$$
\int_{0}^{1} u^{\prime}(x) v^{\prime}(x) \mathrm{d} x-\lambda \int_{0}^{1} u_{*}^{\prime}(x) v_{* u}(x) \mathrm{d} x-\int_{0}^{1} F(u)(x) v(x) \mathrm{d} x=\int_{0}^{1} f(x) v(x) \mathrm{d} x \text { for all } v \in H_{0}^{1}(0,1) .
$$

Theorem 4.2 (Maximum principle). Let $u$ be a solution of (P). If $\lambda \leq 0, a \leq 0$ (resp $\lambda \geq 0, a \geq 0$ ) and $f \geq 0$ (resp $f \leq 0)$, then $u \geq 0$ (resp $u \leq 0)$.

Proof. We set $M(t)=-t_{-}=\min (t, 0)$. Then, $M(u) \in H_{0}^{1}(0,1)$. We obtain (using Sect. 1 and the fact that $a \leq 0)$ :

$$
\int_{\{u \leq 0\}}\left(u^{\prime}\right)^{2}(x) \mathrm{d} x-\lambda \int_{0}^{1} u_{*}^{\prime} M\left(u_{*}\right)=\int_{0}^{1} f M(u)+\int_{0}^{1} F(u)(x) M(u)(x) \mathrm{d} x \leq 0,
$$

that is

$$
\int_{\{u \leq 0\}}\left(u^{\prime}\right)^{2}(x) \mathrm{d} x+\lambda \int_{u_{*}(1)}^{u_{*}(0)} M(t) \mathrm{d} t \leq 0
$$

Since $\lambda \leq 0$, one has:

$$
\int_{\{u \leq 0\}}\left(u^{\prime}\right)^{2}(x) \mathrm{d} x=0=\int_{\inf u}^{\sup u} t_{-} \mathrm{d} t: \inf u \geq 0 .
$$


The proof of the second case (i.e. $\lambda \geq 0, a \geq 0, f \leq 0)$ is the same as above, replacing $-t_{-}$by $t_{+}$. Indeed, in this case, we have the following identity:

$$
\int_{\{u \geq 0\}}\left(u^{\prime}\right)^{2}(x) \mathrm{d} x+\frac{\lambda}{2} u_{+*}^{2}(0)=\int_{0}^{1} f(x) u_{+}(x) \mathrm{d} x+\int_{0}^{1} F(u)(x) u_{+}(x) \mathrm{d} x .
$$

Theorem 4.3. Let $u$ be a solution of $(\mathcal{P})$. Then

i) If $f \neq 0, \lambda a \leq 0$ in $[0,1], u_{-}$has no plateau, that is meas $\left(P\left(u_{-}\right)\right)=0$.

ii) If $\lambda f<0, \lambda a<0$ in $[0,1]$, then the set $\left\{x: u^{\prime}(x)=0\right\}$ is of measure zero.

Proof. If meas $\left(P\left(u_{-}\right)\right) \neq 0$, then there exists a number $t \leq 0$ such that $|u=t|>0$. Since $u \in H^{2}(0,1)$, $u^{\prime \prime}(x)=0=\frac{\mathrm{d}^{+} u_{*}}{\mathrm{~d} s}\left(m_{u}(u(x))\right.$ a.e. $x \in\{u=t\}$. Using the equation satisfied by $u$, one has $f(x)=0$ a.e. on $\{u=t\}$. This contradicts the assumption on $f$.

The proof of the second statement follows a similar idea, i.e. if the measure of the set $\left\{x: u^{\prime}(x)=0\right\}$ is positive, then $u^{\prime \prime}(x)=0$ on that set and then equation $(\mathcal{P})$ leads to:

$$
0 \leq-\lambda^{2} \frac{\mathrm{d}^{+} u_{*}}{\mathrm{~d} s}(\mid u>u(x))-\lambda a(x) F_{0}=\lambda f(x)<0 .
$$

\section{Numerical approximation of the monotone AND RElative REARRAngements OF PIECEWISE LINEAR FUNCTIONS. THE MONODIMENSIONAL CASE}

We begin this section by some results concerning the properties of the monotone and relative rearrangements of piecewise linear continuous functions.

Let $\Delta=\left\{0=x_{0}<x_{1}<\ldots<x_{N+1}=1\right\}$ be a mesh on $[0,1]$. and below $\left\{\varphi_{j}\right\}_{j=0}^{N+1}$ denotes the basis of the piecewise linear functions space relative to $\Delta$, defined by $\varphi_{j}\left(x_{i}\right)=\delta_{i j}$.

The ordered values

$$
\begin{gathered}
\Delta_{u}=\operatorname{sort}\left\{u\left(x_{0}\right), u\left(x_{1}\right), \ldots u\left(x_{N+1}\right)\right\}=\left\{t_{0}=\min u_{j}<t_{1}<\cdots<t_{M}=\max u_{j}\right\} \\
\Delta_{m}=\left\{|\Omega|=m_{0}=m_{u}\left(t_{0}\right) \geq m_{1}=m_{u}\left(t_{1}\right) \geq \cdots \geq m_{M}=m_{u}\left(t_{M}\right)=0\right\}
\end{gathered}
$$

give us the meshes of $I_{u}=\left[\min u_{j}, \max u_{j}\right]$ and $\Omega_{*}=(0,|\Omega|)$, (duplicated values must be suppressed and these meshes may have $M+1<N+2$ points).

Proposition 5.1. Let $u(x)=\sum_{j=0}^{N+1} \alpha_{j} \varphi_{j}$ be a piecewise linear continuous function related to the mesh $\Delta$. Then, the distribution function $m_{u}$ and the decreasing rearrangement are also the piecewise linear functions related to meshes $\Delta_{u}$ and $\Delta_{m}$. Furtheremore,

$$
m_{u}(t)=\left\{\begin{array}{ll}
\frac{m_{i+1} t_{i}-m_{i} t_{i+1}}{t_{i}-t_{i+1}}+\frac{m_{i+1}-m_{i}}{t_{i+1}-t_{i}} t & \text { if } t \in\left[t_{i}, t_{i+1}\right] \quad \text { if } t>t_{M} \\
0 & \text { if } t<t_{0}
\end{array} \quad i=0, \ldots, M-1,\right.
$$




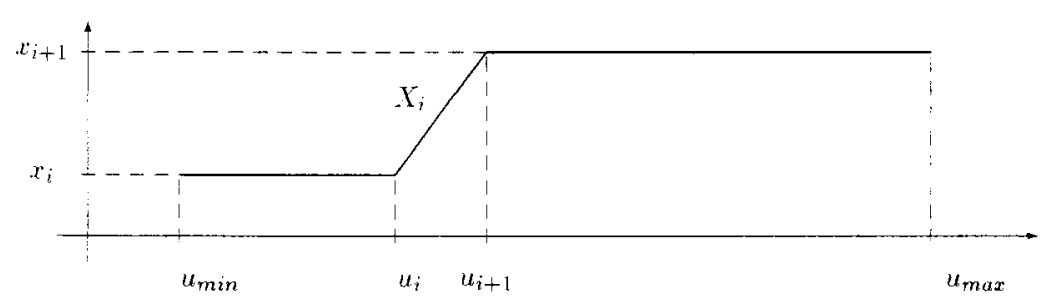

Figure 1. Cases $a_{i}{ }^{1}>0$.

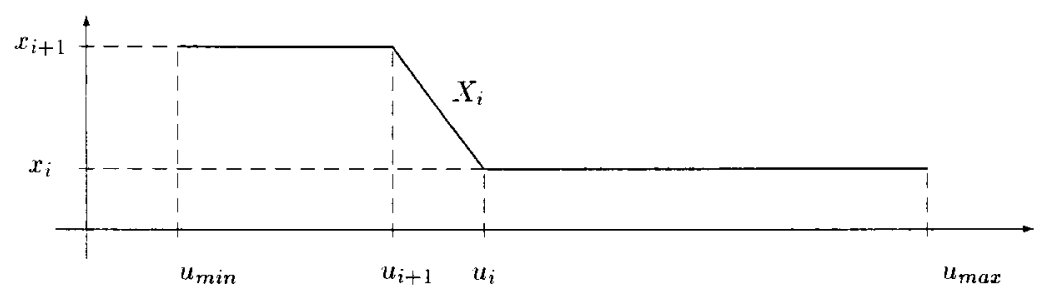

Figure 2. Cases $a_{i}{ }^{1}<0$.

and

$$
u_{*}(s)=\frac{t_{i+1}-t_{i}}{m_{i+1}-m_{i}} s-\frac{m_{i} t_{i+1}-m_{i+1} t_{i}}{m_{i+1}-m_{i}} \quad \text { if } s \in\left[m_{i+1}, m_{i}\right]
$$

Proof. Let $a_{0}^{i}=\frac{u_{i} x_{i+1}-u_{i+1} x_{i}}{x_{i+1}-x_{i}}$ and $a_{1}^{i}=\frac{u_{i+1}-u_{i}}{x_{i+1}-x_{i}}$ be defined on each interval. Let

$$
X_{i}(t)= \begin{cases}x_{i} & \text { if } t>u_{i}, a_{1}^{i}<0 \\ x_{i} & \text { if } t<u_{i}, a_{1}^{i}>0 \\ \frac{t-a_{0}^{i}}{a_{1}^{i}} & \text { if } t \in\left(\min \left\{u_{i}, u_{i+1}\right\}, \max \left\{u_{i}, u_{i+1}\right\}\right)=I_{u_{i}} \\ x_{i+1} & \text { if } t>u_{i+1}, a_{1}^{i}>0 \\ x_{i+1} & \text { if } t<u_{i+1}, a_{1}^{i}<0\end{cases}
$$

be the extended inverse of $\left.u\right|_{\left[x_{i}, x_{i+1}\right]}$ showed in Figures $1-2$ if $a_{1}^{i} \neq 0$.

The measure can be exactly computed by:

$$
m_{u}\left(t^{+}\right)=\sum_{\substack{j \in \mathcal{N}(t) \\ a_{1}^{j}>0}}\left[x_{j+1}-X_{j}(t)\right]+\sum_{\substack{j \in \mathcal{N}(t) \\ a_{1}^{j}<0}}\left[X_{j}(t)-x_{j}\right]+\sum_{\substack{j \in \mathcal{N}(t) \\ a_{1}^{j}=0}}\left[x_{j+1}-x_{j}\right]
$$

where $\mathcal{N}(t)=\left\{i: t<\max \left\{u_{i}, u_{i+1}\right\}\right\}$. Since the functions $X_{i}$ are piecewise linear continuous, it is the same for $m_{u}$ if $u$ has not plateau. In another case, $\left|u=t_{i}\right|>0$ is the jump at the value $t_{i}$. 
Remark 5.1. Derivatives of $u_{*}$ (respectively $m_{u}$ ) are not defined at the mesh points but they are constant on each interval $\left(m_{i+1}, m_{i}\right)$ (repectively $\left.\left(t_{i}, t_{i+1}\right)\right)$. Inside each interval we have:

$$
m_{u}^{\prime}(t)=\sum_{\substack{j \in \mathcal{N}(t) \\ a_{1}^{j}>0}}-X_{j}^{\prime}(t)+\sum_{\substack{j \in \mathcal{N}(t) \\ a_{1}^{j}<0}} X_{j}^{\prime}(t)=\sum_{j \in \mathcal{N}_{*}(t)}-\operatorname{sig}\left(a_{1}^{j}\right) \frac{x_{j+1}-x_{j}}{u_{j+1}-u_{j}}
$$

where $\mathcal{N}_{*}(t)=\left\{i: \min \left\{u_{i}, u_{i+1}\right\}<t<\max \left\{u_{i}, u_{i+1}\right\}\right\}$, if $t \neq u_{j}$ for all $j=0,1, \ldots N+1$.

Proposition 5.2. If $b$ is a piecewise linear positive function, $m_{u}^{b}(t)=\int_{\{x: u(x)>t\}} b(x) \mathrm{d} x$ is a piecewise quadratic function related to mesh $\Delta_{u}$.

Proof. As in the previous proposition, we find the $b$-weighted measure

$$
\begin{aligned}
m_{u}^{b}\left(t^{+}\right) & =\sum_{\substack{j \in \mathcal{N}(t) \\
a_{1}^{j}>0}} \int_{X_{j}(t)}^{x_{j+1}} b(x) \mathrm{d} x+\sum_{\substack{j \in \mathcal{N}(t) \\
a_{1}^{j}<0}} \int_{x_{j}}^{X_{j}(t)} b(x) \mathrm{d} x+\sum_{\substack{j \in \mathcal{N}(t) \\
a_{1}^{j}=0}} \int_{x_{j}}^{x_{j+1}} b(x) \mathrm{d} x \\
& =\sum_{\substack{j \in \mathcal{N}(t) \\
a_{1}^{j}=0}}\left[g\left(x_{j+1}\right)-g\left(X_{j}(t)\right)\right]+\sum_{\substack{j \in \mathcal{N}(t) \\
a_{1}^{j}<0}}\left[g\left(X_{j}(t)\right)-g\left(x_{j}\right)\right]+\sum_{\substack{j \in \mathcal{N}(t) \\
a_{1}^{j}=0}} g\left(x_{j+1}\right)-g\left(x_{j}\right)
\end{aligned}
$$

where $g$ is a primitive of $b$, which is a quadratic function.

Theorem 5.1. Let $b$ and $u$ be piecewise linear functions, where $u$ has not plateau. Then the relative rearrangement is a piecewise linear function.

Proof. Following Propositions 5.1 and 5.2, it suffices, in order to compute the $b$-measures, to solve the linear system:

$$
\left.\begin{array}{l}
\alpha_{i} t_{i}^{2}+\beta_{i} t_{i}+\gamma_{i}=m_{u}^{b}\left(t_{i}\right) \\
\alpha_{i} t_{i+\frac{1}{2}}^{2}+\beta_{i} t_{i+\frac{1}{2}}+\gamma_{i}=m_{u}^{b}\left(t_{i+\frac{1}{2}}\right) \\
\alpha_{i} t_{i+1}^{2}+\beta_{i} t_{i+1}+\gamma_{i}=m_{u}^{b}\left(t_{i+1}\right)
\end{array}\right\}
$$

at extremities and at the middle point, $t_{i+\frac{1}{2}}$, in the interval $\left[t_{i}, t_{i+1}\right]$. Consequently, the relative rearrangement is a piecewise linear function inside each interval $\left(m_{i+1}, m_{i}\right)$ :

$$
b_{* u}(s)=\frac{m_{u}^{b^{\prime}}\left(u_{*}(s)\right)}{m_{u}^{\prime}\left(u_{*}(s)\right)}=\frac{2 \alpha_{i} u_{*}(s)+\beta_{i}}{\frac{m_{i+1}-m_{i}}{t_{i+1}-t_{i}}}
$$

where the coefficients $\alpha_{i}, \beta_{i}$ are given on each interval $\left(t_{i}, t_{i+1}\right)$ by

$$
\begin{aligned}
\alpha_{i} & =\frac{2}{\Delta t_{i}^{2}}\left(m_{i+1}^{b}-2 m_{i+\frac{1}{2}}^{b}+m_{i}^{b}\right) \\
\beta_{i} & =\frac{m_{i+1}^{b}-m_{i}^{b}}{\Delta t_{i}}-\frac{2}{\Delta t_{i}^{2}}\left(m_{i+1}^{b}-2 m_{i+\frac{1}{2}}^{b}+m_{i}^{b}\right)\left(t_{i+1}+t_{i}\right)
\end{aligned}
$$

whith $\Delta t_{i}=t_{i+1}-t_{i}$. 
Remark 5.2. If $b$ is continuous and $u$ is monotone, $\mathcal{N}_{*}(t)$ (see Rem. 4.1) must a unique element, and the relative rearrangement is continuous. But, in another case, there are two or more elements in the set $\mathcal{N}_{*}(t)$, and the continuity can not be assured as we will show in the example below.

Example. Let $\Omega=\left(0, \frac{5}{2}\right), \Omega_{*}=(0,|\Omega|)=\left(0, \frac{5}{2}\right), b(x)=3 x$ and

$$
u(x)= \begin{cases}x & 0 \leq x \leq 1 \\ \frac{1}{2} x+\frac{1}{2} & 1<x \leq 2 \\ \frac{7}{2}-x & 2<x \leq \frac{5}{2}\end{cases}
$$

In this case

$$
b_{* u}(s)= \begin{cases}6-s & 0 \leq s<\frac{3}{2} \\ 3\left(\frac{5}{2}-s\right) & \frac{3}{2} \leq s \leq \frac{5}{2}\end{cases}
$$

which is not continuous at $\frac{3}{2}$.

\section{Numerical SOlUtion OF THE VARIATIONAL NONLOCAL PROBLEM}

We shall begin by solving the variational problem $\left(\mathcal{P}_{v}\right)$ in the case $a(x)=0$. If $f$ is a symmetric function and we take a suitable mesh, the discrete problem turns into a linear system with tridiagonal matrix but, in more general cases, the nonlocal terms will be treated by a fixed point algorithm.

In order to find a numerical solution we consider the usual $\mathbb{P}_{1}$ finite element approach.

Let $\Delta=\left\{0=x_{0}<x_{1}<\cdots<x_{N+1}=1\right\}$ be a mesh in the interval $[0,1]$. We consider the space $V_{h}=$ $\left\{v \in C^{0}[0,1]: v(0)=v(1)=0,\left.v\right|_{\left[x_{i}, x_{i+1}\right]} \in \mathbb{P}_{1}\right.$, for all $\left.i=0,1, \ldots N\right\}$. Thus, the discrete variational problem reads:

$$
\left(\mathcal{P}_{v 0}^{h}\right)\left\{\begin{array}{l}
\text { Find } u_{h} \in V_{h} \text { such that } \\
\int_{0}^{1} u_{h}^{\prime}(x) v_{h}^{\prime}(x) \mathrm{d} x-\lambda \int_{0}^{1} u_{h *}^{\prime}(\sigma)\left(v_{h}\right)_{* u_{h}}(\sigma) \mathrm{d} \sigma-\int_{0}^{1} f(x) v_{h}(x) \mathrm{d} x=0 \text { for all } v_{h} \in V_{h} .
\end{array}\right.
$$

We are looking for a solution of $\left(\mathcal{P}_{v 0}^{h}\right)$ without plateau. In that case, by using previous results, we obtain

$$
\begin{aligned}
\int_{0}^{1} u_{h *}^{\prime}(\sigma)\left(v_{h}\right)_{* u_{h}}(\sigma) \mathrm{d} \sigma & =\sum_{i=0}^{M-1} \int_{m_{i+1}}^{m_{i}} u_{h *}^{\prime}(\sigma)\left(2 \alpha_{i} u_{h *}(\sigma)+\beta_{i}\right)\left(\frac{t_{i+1}-t_{i}}{m_{i+1}-m_{i}}\right) \mathrm{d} \sigma \\
& =\sum_{i=0}^{M-1}\left(v_{h}\right)_{* u_{h}}\left(m_{i+\frac{1}{2}}\right)\left(u_{h *}\left(m_{i}\right)-u_{h *}\left(m_{i+1}\right)\right) .
\end{aligned}
$$

As $\left(v_{h}\right)_{* u}$ is continuous on each interval, values $\left(v_{h}\right)_{* u_{h}}\left(m_{i+\frac{1}{2}}\right)$ are well defined. Thus, the variational formulation can be rewritten as:

$$
\left\{\begin{array}{l}
\{\text { Find }\} \mathrm{u}_{\mathrm{h}} \in \mathrm{V}_{\mathrm{h}} \text { such that } \\
\int_{0}^{1} u_{h}^{\prime}(x) v_{h}^{\prime}(x) \mathrm{d} x-\lambda \sum_{i=1}^{M-1}\left(v_{h}\right)_{* u_{h}}\left(m_{i+\frac{1}{2}}\right)\left(u_{h *}\left(m_{i}\right)-u_{h *}\left(m_{i+1}\right)\right)-\int_{0}^{1} f(x) v_{h}(x) \mathrm{d} x=0 \\
\text { for all } v_{h} \in V_{h} .
\end{array}\right.
$$


The previous variational problem amounts to solving a finite dimensional system associated with a basis $\left\{\varphi_{k}\right\}_{k=1}^{N}$ of $V_{h}$ :

$$
\left(\mathcal{P}_{v 0}^{h}\right)^{\prime}\left\{\begin{array}{l}
\text { Find } u_{h}=\sum_{k=1}^{N} u_{k} \varphi_{k} \in V_{h} \text { such that } \\
\int_{0}^{1} u_{h}^{\prime}(x) \varphi_{k}^{\prime}(x) \mathrm{d} x-\lambda \sum_{i=0}^{M-1}\left(\varphi_{k}\right)_{* u_{h}}\left(m_{i+\frac{1}{2}}\right)\left(u_{h *}\left(m_{i}\right)-u_{h *}\left(m_{i+1}\right)\right)-\int_{0}^{1} f(x) \varphi_{k}(x) \mathrm{d} x=0 \\
\text { for all } k=1,2, \ldots N
\end{array}\right.
$$

The key of this result is the linearity in the test function $v_{h}$ proved in Lemma 2.4.

Values $m_{i}$ and $\left(\varphi_{k}\right)_{* u}\left(m_{i+\frac{1}{2}}\right)$ can be easily computed by using the mesh values $u_{i}$ and a suitable function $\eta:\{0,1,2, \ldots N+1\} \rightarrow\{0,1,2, \ldots M\}$ such that $t_{\eta(i)}=u_{i}(\eta$ is well defined since there are not repeated values $t$ ).

\subsection{The symmetric case}

The biggest difficults to write the nonlinear system $\left(\mathcal{P}_{v 0}^{h}\right)^{\prime}$ is the explicit expression of the measures and rearrangements as function of the values $\left\{u_{0}, u_{1}, u_{2}, \ldots u_{N}, u_{N+1}\right\}$, the mesh $\left\{x_{0}, x_{1}, x_{2}, \ldots x_{N}, x_{N+1}\right\}$ and the permutation $\eta$. Even though, we can compute them by using formulas (23-24-28-29-30), we shall have "nice" equations only in very particular cases.

Thanks to the qualitative properties of the solution, if $f$ is a positive symmetric function $(f(x)=f(1-x))$, we can suppose that $u$ has not plateau and that it is positive, symmetric respect to the middle point in the interval $(0,1)$ (i.e. $u(x)=u(1-x)$ ), which is its unique maximum, and the minimum is reached on the boundary. Furthermore, we are going to consider a spatial mesh $\Delta\left\{0=x_{0}<x_{1}<\cdots<x_{N+1}=1\right\}$ with equidistant points, (i.e. $h=x_{i}-x_{i-1}$ for all $i=1, \ldots N+1$ ) and $N$ odd (so, the middle point of the interval is $x_{\frac{N+1}{2}}$ and the discrete solution has no plateau). Then, $M=\frac{N+1}{2}$ and

$$
\eta(i)=\left\{\begin{array}{ll}
i & 0 \leq i \leq \frac{N+1}{2} \\
N-i+1 & \frac{N+1}{2} \leq i \leq N+1
\end{array} .\right.
$$

Now, the relative rearrangements of the piecewise linear basis related to mesh $\Delta$ can be exactly computed. More precisely we have

Proposition 6.1. $\quad$ i) If $1 \leq i<\frac{N+1}{2}$, then

$$
\varphi_{i * u}(s)= \begin{cases}\frac{X_{i-1}\left(u_{*}(s)\right)-x_{i-1}}{2 h} & \text { if } s \in\left(m_{i}, m_{i-1}\right) \\ -\frac{X_{i}\left(u_{*}(s)\right)-x_{i+1}}{2 h} & \text { if } s \in\left(m_{i+1}, m_{i}\right) \\ 0 & \text { otherwise. }\end{cases}
$$


ii) If $\frac{N+1}{2}<i \leq N$, then

$$
\varphi_{i * u}(s)= \begin{cases}-\frac{X_{i}\left(u_{*}(s)\right)-x_{i+1}}{2 h} & \text { if } s \in\left(m_{\eta(i)}, m_{\eta(i+1)}\right) \\ \frac{X_{i-1}\left(u_{*}(s)\right)-x_{i-1}}{2 h} & \text { if } s \in\left(m_{\eta(i-1)}, m_{\eta(i)}\right) \\ 0 & \text { otherwise. }\end{cases}
$$

iii) If $i=\frac{N+1}{2}$, then

$$
\varphi_{i * u}(s)= \begin{cases}\frac{X_{i-1}\left(u_{*}(s)\right)-x_{i-1}}{h} & \text { if } s \in\left(m_{i}, m_{i-1}\right) \\ 0 & \text { otherwise. }\end{cases}
$$

By using this expressions in $\left(\mathcal{P}_{v 0}^{h}\right)^{\prime}$ we obtain the linear problem

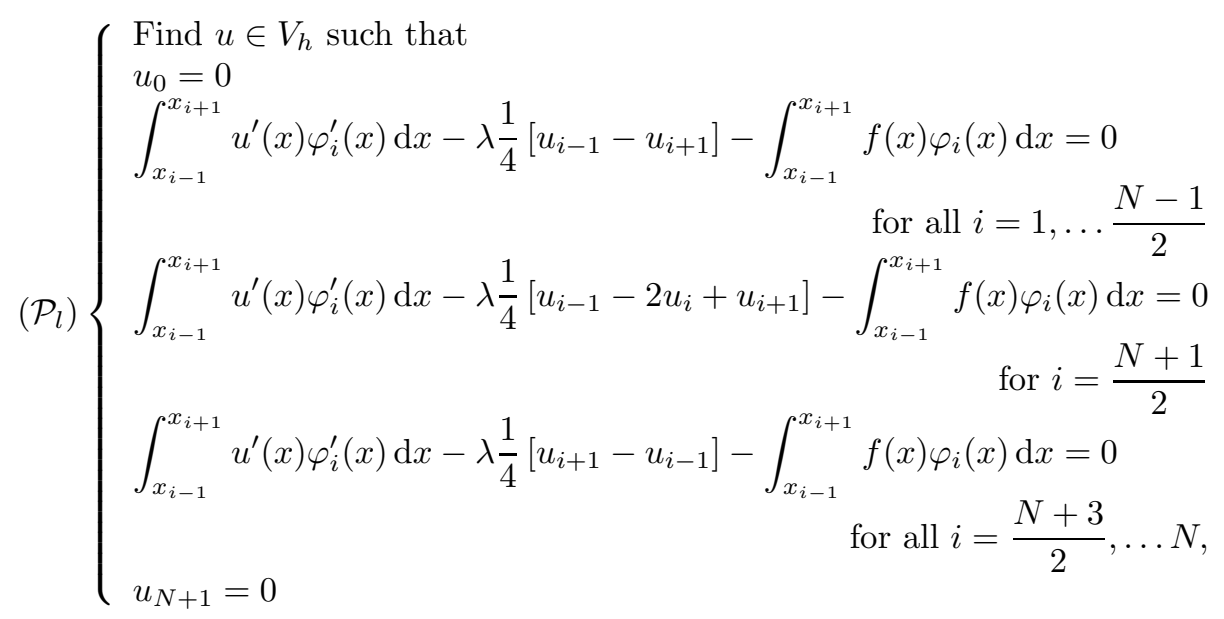

which can be reduced to a linear system whose matrix reads

$$
\mathcal{A}_{h}=\left(\begin{array}{cccc|c|cccc}
\frac{2}{h} & -\frac{1}{h}+\frac{\lambda}{4} & & & 0 & 0 & \ldots & 0 & 0 \\
-\frac{1}{h}-\frac{\lambda}{4} & \frac{2}{h} & -\frac{1}{h}+\frac{\lambda}{4} & & 0 & 0 & \ldots & 0 & 0 \\
& \ddots & \ddots & \ddots & \vdots & \vdots & & \vdots & \vdots \\
& & -\frac{1}{h}-\frac{\lambda}{4} & \frac{2}{h} & -\frac{1}{h}+\frac{\lambda}{4} & 0 & \ldots & 0 & 0 \\
\hline 0 & \ldots & 0 & -\frac{1}{h}-\frac{\lambda}{4} & \frac{2}{h}+\frac{\lambda}{2} & -\frac{1}{h}-\frac{\lambda}{4} & \ldots & 0 & 0 \\
\hline 0 & \cdots & 0 & 0 & -\frac{1}{h}+\frac{\lambda}{4} & \frac{2}{h} & -\frac{1}{h}-\frac{\lambda}{4} & & \\
\vdots & & \vdots & \vdots & \vdots & \ddots & \ddots & \ddots & \\
0 & \ldots & 0 & 0 & 0 & & -\frac{1}{h}+\frac{\lambda}{4} & \frac{2}{h} & -\frac{1}{h}-\frac{\lambda}{4} \\
0 & \cdots & 0 & 0 & 0 & & & -\frac{1}{h}+\frac{\lambda}{4} & \frac{2}{h}
\end{array}\right)
$$

This matrix is a $M$-matrix if $0<\lambda<\frac{4}{h}$ (see [61]) and satisfies a discrete maximum principle (if $\mathbf{b}>\mathbf{0}$, then the solution of $\mathcal{A}_{h} \mathbf{u}=\mathbf{b}$ satisfies $\mathbf{u}>\mathbf{0}$ ) according to the results for the continuous problem. 


\subsection{The nonsymmetric case}

When $f$ is not symmetric, we cannot expect a symmetric solution. Then, the nonlinear system $\left(\mathcal{P}_{v 0}^{h}\right)^{\prime}$ is solved by using a fixed point algorithm:

- Given $u_{h}^{(0)} \in \hat{V}_{h}=\left\{v \in C^{0}[0,1]: v(0)=c_{0}, v(1)=c_{1},\left.v\right|_{\left[x_{i}, x_{i+1}\right]} \in \mathbb{P}_{1}\right.$, for all $\left.i=0,1, \ldots N\right\}$, for $l=$ $1,2, \ldots$, we find $u_{h}^{(l)}=c_{0} \varphi_{0}+\sum_{k=1}^{N} u_{k}^{(l)} \varphi_{k}+c_{1} \varphi_{N+1} \in \hat{V}_{h}$ such that

$$
\left\{\begin{array}{l}
\int_{0}^{1}\left(u_{h}^{(l)}\right)^{\prime}(x) \varphi_{k}^{\prime}(x) \mathrm{d} x= \\
\lambda \sum_{i=0}^{M-1}\left(\varphi_{k}\right)_{* u_{h}^{(l-1)}}\left(m_{i+\frac{1}{2}}^{(l-1)}\right)\left(u_{h *}^{(l-1)}\left(m_{i}^{(l-1)}\right)-u_{h *}^{(l-1)}\left(m_{i+1}^{(l-1)}\right)\right)+\int_{0}^{1} f(x) \varphi_{k}(x) \mathrm{d} x \\
\quad \text { for all } k=1,2, \ldots N .
\end{array}\right.
$$

In order to compute relative and decreasing rearrangements of $\varphi_{k}$ and $u_{h}^{(l-1)}$ in each iteration, we must find the table (the index $(l-1)$ is drooped)

\begin{tabular}{||c|c|c|c||}
\hline $\mathbf{t}$ & $\mathbf{m}$ & $\beta^{k}$ & $\Phi^{k}$ \\
\hline \hline$t_{0}$ & $m_{0}$ & $\beta_{0}^{k}$ & $\phi_{0}^{k}$ \\
\hline$t_{1}$ & $m_{1}$ & $\beta_{1}^{k}$ & $\phi_{1}^{k}$ \\
\hline$\vdots$ & $\vdots$ & $\vdots$ & $\vdots$ \\
\hline$t_{M}$ & $m_{M}$ & $\beta_{M}^{k}$ & $\phi_{M}^{k}$ \\
\hline
\end{tabular}

$$
\beta_{i}^{k}=\int_{\left\{u_{h}^{(l-1)}>u_{h *}^{(l-1)}\left(m_{i}\right)\right\}} \varphi_{k}(x) \mathrm{d} x \quad \phi_{i}^{k}=\frac{\beta_{i+1}^{k}-\beta_{i}^{k}}{m_{i+1}-m_{i}} i=0, \ldots M-1
$$

where the two final columns correspond to a generic basis function $\varphi_{k}$.

The measures $\beta_{i}^{k}$ are exactly computed by a numerical quadrature formula of low order (for instance the middle point or the trapezoidal rules). Furthermore, if $u_{h}^{(l-1)}$ is without flat regions, the measures are continuous functions and $m_{i}=m_{u_{h}^{(l-1)}}\left(t_{i}\right), \beta_{i}^{k}=m_{u_{h}^{(l-1)}}^{\varphi_{k}}\left(t_{i}\right)$. So, from $(28)$, we have $\phi_{i}^{k}=\left(\varphi_{k}\right)_{* u_{h}^{(l-1)}}\left(\frac{m_{i}+m_{i+1}}{2}\right)$. Finally, we have $u_{h *}^{(l-1)}\left(m_{i}\right)=t_{i}$ and the rightside in (31) can be evaluated.

The same technique works for solving the full problem:

$$
\left(\mathcal{P}_{v}^{h}\right)^{\prime}\left\{\begin{array}{l}
\text { Find } u_{h}=\sum_{k=1}^{N} u_{k} \varphi_{k} \in V_{h} \text { such that } \\
\int_{0}^{1} u_{h}^{\prime}(x) \varphi_{k}^{\prime}(x) \mathrm{d} x-\lambda \sum_{i=0}^{M-1}\left(\varphi_{k}\right)_{* u_{h}}\left(m_{i+\frac{1}{2}}\right)\left(u_{h *}\left(m_{i}\right)-u_{h *}\left(m_{i+1}\right)\right)-\int_{0}^{1} F\left(u_{h}\right)(x) \varphi_{k}(x) \mathrm{d} x= \\
=\int_{0}^{1} f(x) \varphi_{k}(x) \mathrm{d} x \quad \text { for all } k=1,2, \ldots N
\end{array}\right.
$$

where $F\left(u_{h}\right)(x)=a(x)\left[F_{0}^{2}-\int_{m_{u_{h}}(0)}^{m_{u_{h}}\left(u_{h+}(x)\right)} p^{\prime}\left(u_{h *}\right)(s) u_{h *}^{\prime}(s) b_{* u_{h}}(s) \mathrm{d} s\right]_{+}^{1 / 2}$. 
For each fixed point iteration we must find $u^{(l)} \in \hat{V}_{h}$ the solution of

$$
\left\{\begin{array}{l}
\int_{0}^{1}\left(u_{h}^{(l)}\right)^{\prime}(x) \varphi_{k}^{\prime}(x) \mathrm{d} x=\int_{0}^{1} F\left(u_{h}^{(l-1)}\right)(x) \varphi_{k}(x) \mathrm{d} x+ \\
+\lambda \sum_{i=0}^{M-1}\left(\varphi_{k}\right)_{* u_{h}^{(l-1)}}\left(m_{i+\frac{1}{2}}^{(l-1)}\right)\left(u_{h *}^{(l-1)}\left(m_{i}^{(l-1)}\right)-u_{h *}^{(l-1)}\left(m_{i+1}^{(l-1)}\right)\right)+\int_{0}^{1} f(x) \varphi_{k}(x) \mathrm{d} x \\
\quad \text { for all } k=1,2, \ldots N .
\end{array}\right.
$$

The relative rearrangement $b_{* u_{h}^{(l-1)}}$ in the new term $F\left(u_{h}^{(l-1)}\right)$ can be approached inside the intervals $\left[m_{i+1}, m_{i}\right]$ by $w_{i}$ where

$$
w_{i}=\frac{m_{i+1}^{b}-m_{i}^{b}}{m_{i+1}-m_{i}}, \quad \quad m_{i}^{b}=\int_{\left\{u_{h}^{(l-1)}>u_{h *}^{(l-1)}\left(m_{i}\right)\right\}} b(x) \mathrm{d} x, \quad i=0, \ldots M-1,
$$

just by adding two columns in the above table.

\subsection{Numerical results}

Firstly, we have tested the accuracy and the influence of the mesh on several problems for the simplified model $(a(x)=0)$. More precisely, given a data function $f$, we compute the solution $u$ of

$$
\begin{cases}-u^{\prime \prime}(x)-\lambda u_{*}^{\prime}\left(m_{u}(u(x))\right)=f(x) \text { in }(0,1) \\ u(0)=c_{0} \quad u(1)=c_{1}\end{cases}
$$

in the cases:

Test 1: $f(x)=\pi^{2} \sin (\pi x)+\frac{\lambda \pi}{2}|\cos (\pi x)|$ (the exact solution is $u(x)=\sin (\pi x)$ ) and,

Test 2: $f(x)=2+\frac{\lambda}{2} \sqrt{1-4 x(1-x)}$ (the exact solution is $u(x)=x(1-x)$ ).

These problems have been solved by using five meshes indexed by their number of points, $N$, in view of to see the influence of the nonlocal term in the approximation order.

Table 1. Errors in $L^{\infty}$-norm.

\begin{tabular}{||c||c|c|c|c|c|c||}
\hline$N$ & 11 & 21 & 31 & 41 & 81 & 91 \\
\hline \hline Test 1 & $4.28 \times 10^{-3}$ & $1.08 \times 10^{-3}$ & $4.82 \times 10^{-4}$ & $2.71 \times 10^{-4}$ & $6.76 \times 10^{-5}$ & $5.35 \times 10^{-5}$ \\
\hline Test 2 & $1.11 \times 10^{-3}$ & $2.76 \times 10^{-4}$ & $1.23 \times 10^{-4}$ & $6.91 \times 10^{-5}$ & $1.73 \times 10^{-5}$ & $1.36 \times 10^{-5}$ \\
\hline
\end{tabular}

The table 1 shows the same approximation order as in the linear problem (Test 1) but there is not exactly computation when the solution belongs to $\mathbb{P}_{2}$ (Test 2).

Next, a more general case is considered, where $f$ and, consequently, the solution $u$ are nonsymmetric:

Test 3: $f(x)=-2-\lambda\left\{\begin{array}{ll}\frac{4(1-\sqrt{0.25+4 x(x-0.5)})-3}{2} & \text { if } x \in(0,0.5) \\ \frac{-1-2 \sqrt{0.25+4 x(x-0.5)}}{8} & \text { if } x \in(0.5,1) .\end{array}, u(x)=x(x-0.5)\right.$. 


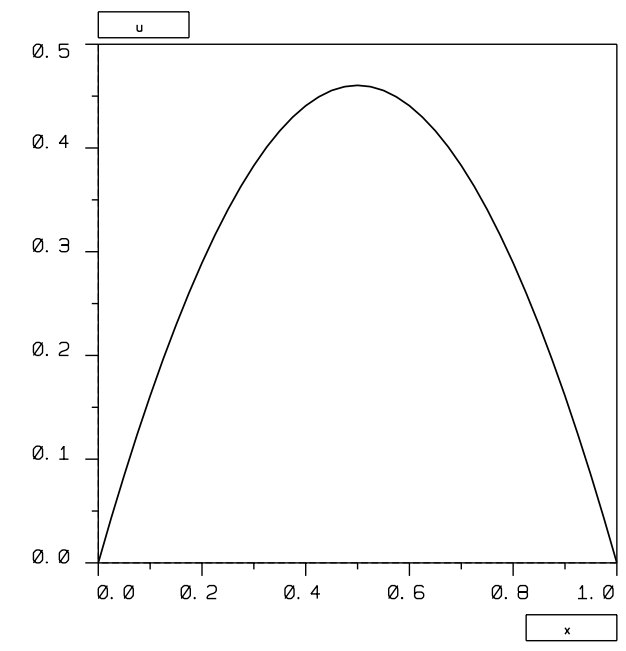

FIGURE 3. Solution.

Now, the analyse in Section 6.1 is no more valid, but the difficulty coming from the nonlocal terms can be solved by using the fixed point method. Errors in $L^{\infty}$-norm are repported here below.

\begin{tabular}{||c||c|c|c|c|c|c||}
\hline$N$ & 11 & 21 & 31 & 41 & 81 & 91 \\
\hline Test 3 & $2.40 \times 10^{-3}$ & $1.18 \times 10^{-3}$ & $9.34 \times 10^{-4}$ & $6.72 \times 10^{-4}$ & $3.56 \times 10^{-4}$ & $3.26 \times 10^{-4}$ \\
\hline
\end{tabular}

In order to test the influence of the approximation of the relative rearrangement of a given function $b$, we take $f(x)=\pi^{2} \cos (\pi x)-\lambda g(\cos (\pi x)) \sin (\pi x), b(x)=\sin (\pi x)$ and we solve

$$
\left\{\begin{array}{l}
-u^{\prime \prime}(x)-\lambda g(u(x)) b_{* u}\left(m_{u}(u(x))\right)=f(x) \text { in }(0,1) \\
u(0)=c_{0} \quad u(1)=c_{1}
\end{array}\right.
$$

The convergence of fixed point iterations is not assured since the relative rearrangement does not satisfy a Lipschitz condition related to $u$. Nevertheless, in some particular cases, it can be proved that

$$
\left\|b_{* u}-b_{* v}\right\| \leq L(b)\|u-v\|
$$

(see [46] for details).

We pose $g(u)=u$ in $\underline{\text { Test } 4}$ and $g(u)=u^{2}$ in $\underline{\text { Test } 5}$ (in both cases the exact solution is $u(x)=\cos (\pi x)$ ) and we find the same order of approximation as in the linear problem:

\begin{tabular}{||c||c|c|c|c|c|c||}
\hline$N$ & 11 & 21 & 31 & 41 & 81 & 91 \\
\hline \hline Test 4 & $8.40 \times 10^{-4}$ & $2.10 \times 10^{-4}$ & $9.33 \times 10^{-5}$ & $5.26 \times 10^{-5}$ & $1.32 \times 10^{-5}$ & $1.04 \times 10^{-5}$ \\
\hline Test 5 & $2.77 \times 10^{-3}$ & $6.93 \times 10^{-4}$ & $3.10 \times 10^{-4}$ & $1.74 \times 10^{-4}$ & $4.36 \times 10^{-5}$ & $3.44 \times 10^{-5}$ \\
\hline
\end{tabular}

Finally, we solve $\left(\mathcal{P}_{v}\right)$ with $f(x)=0, b(x)=x^{2}, a(x)=1, p(x)=\frac{x^{2}}{2}$ and $F_{0}=4$. The computed solution is given in Figure 3.

Remark 6.1. Analogous discretization and approximation techniques can be employed for $2-D$ and $3-D$ nonsymmetric problems $($ see $[5]$ for $(\mathcal{P S})$ ). 
Acknowledgements. We wish to thank A. Miranville for having corrected the English.

\section{REFERENCES}

[1] F. Almgren and E. Lieb, Symmetric rearrangement is sometimes continuous. J. Amer. Math. Soc. 2 (1989) 683-772.

[2] E. Beretta and M. Vogelius, Symmetric rearrangement is sometimes continuous, An inverse problem originating from Magnetohydrodynamics II: the case of the Grad-Shafranov equation. Indiana University Mathematics Journal 41 (1992) $1081-1117$.

[3] H. Berestycki and H. Brezis, On a free boundary problem arising in plasma physics. Nonlinear Anal. 4 (1980) 415-436.

[4] A. Bermúdez and C. Moreno, Duality methods for solving variational inequalities. Comp. and Math. Appl. 7 (1981) $43-58$.

[5] A. Bermúdez and M.L. Seoane, Numerical Solution of a Nonlocal Problem Arising in Plasma Physics. Mathematical and Computing Modelling. 27 (1998) 45-59.

[6] J. Blum, Numerical Simulation and Optimal Control in Plasma Physics, Wiley, Gauthier-Villars (1989).

[7] J. Blum, T. Gallouët and J. Simon, Existence and Control of plasma equilibrium in a tokamak. SIAM J. Math. Anal. 17 (1986) 1158-1177.

[8] A.H. Boozer, Establishment of magnetic coordinates for given magnetic field. Phys. Fluids 25 (1982) 520-521.

[9] H. Brezis, Opérateurs maximaux monotones et semigroupes de contractions dans les espaces de Hilbert, North-Holland (1973).

[10] G. Chiti, Rearrangements of functions and convergence in Orlicz spaces. Applicable Analysis 9 (1979).

[11] K.M. Chong and N.M. Rice, Equimesurable rearrangements of functions, Queen's University (1971).

[12] P.G. Ciarlet, Introduction to Numerical Linear Algebra and Optimization, Cambrigde University Press (1989).

[13] J.M. Coron, The Continuity of the Rearrangement in $W^{1, p}(\mathbb{R})$. Annali della Scuola Normale Superiore di Pisa. Série IV 11 (1984) $57-85$.

[14] R. Courant and D. Hilbert, Methods of Mathematical Physics, vol. I., Interscience Pub. (1953).

[15] J.I. Díaz, Modelos bidimensionales de equilibrio magnetohidrodinámico para Stellarators. Formulación global de las ecuacion es diferenciales no lineales y de las condiciones de contorno, CIEMAT, Informe \#1 (1991).

[16] J.I. Díaz, Modelos bidimensionales de equilibrio magnetohidrodinámico para Stellarators. Resultados de existencia de soluciones, CIEMAT, Informe \#2 (1992).

[17] J.I. Díaz, Modelos bidimensionales de equilibrio magnetohidrodinámico para Stellarators. Multiplicidad y dependencia de parámetros, CIEMAT, Informe \#3 (1993).

[18] J.I. Díaz and J.M.Rakotoson, On a two-dimensional stationary free boundary problem arising in the confinement of a plasma in a Stellarator. C.R. Acad. Sci. Paris Serie I 317 (1993) 353-358.

[19] J.I. Díaz and J.M. Rakotoson, On a nonlocal stationary free boundary problem arising in the confinement of a plasma in a Stellarator geometry. Arch. Rat. Mech. Anal. 134 (1996) 53-95.

[20] I.Ekeland and R. Temam, Convex Analysis and Variational Problems, North-Holland (1976).

[21] E. Fernández-Cara and C. Moreno, Critical Point Approximation Through Exact Regularization. Math. Comp. 50 (1988) 139-153.

[22] J.P. Freidberg, Ideal Magnetohydrodynamics, Plenum Press (1987).

[23] A. Friedman, Variational principles and free-boundary problems, John Wiley and Sons (1982).

[24] R. Glowinski, Numerical methods for non linear variational problems, Springer Verlag (1984).

[25] H. Grad, Mathematical problem arising in plasmas physics. Proc. Intern. Congr. Math. Nice (1970).

[26] J.M. Greene and J.L. Johnson, Determination of Hydromagnetic Equilibria. Phys. Fluids 27 (1984) 2101-2120

[27] G.H. Hardy, J.E. Littlewood and G. Polya, Inequalities, Cambridge University Press (1964).

[28] T.C. Hender and B.A. Carreras, Equilibrium calculation for helical axis Stellarators. Phys. Fluids 27 (1984) $2101-2120$.

[29] B.Heron and M.Sermange, Non convex methods for computing free boundary equilibria of axially symmetric plasmas, Rapport de Recherche, I.N.R.I.A. (1981).

[30] M.D. Kruskal and R.M. Kulsrud, Equilibrium of Magnetically Confined Plasma in a Toriod. Physics of Fluids 1, No. 4, (1958) 265-274.

[31] A. Marrocco and O. Pironneau, Optimum desing with lagrangian finite elements: desing of an electromagnet, Rapport de Recherche, I.N.R.I.A. (1977).

[32] F. Mignot and J.P. Puel, On a class of nonlinear problems with positive, increasing, convex nonlinearity. Comm. Par. Diff. Eq. 5 (1980) 791-836.

[33] J. Mossin and J.M. Rakotoso, Isoperimetric inequalities in parabolic equations. Annali della Scuola Normale Superiore di Pisa. Série IV 13, No. 1, (1986) 51-73.

[34] J. Mossino and R. Temam, Directional Derivative of the Increasing Rearrangement Mapping and Application to a Queer Differential Equation in Plasma Physics. Duke Mathematical Journal 48 (1981) 475-495.

[35] J. Mossino and R. Temam, Free boundary problems in plasma physics, review of results and new developments. Free Boundary Problems: theory and applications. Vol I-II. Proc. Montec atini Symposium (1981). A. Fasano and M. Primicerio Eds, Pitman (1983) 672-681.

[36] J. Mossino, Inégalités isopérmétriques et applications en physique, Hermann (1984).

[37] K. Miyamoto, Plasma Physics for Nuclear Fusion, The M.I.T. Press (1987). 
[38] J.F. Padial, EDPs no lineales originadas en plasmas de fusión y filtración en medios porosos, Thesis Doctoral, Universidad Complutense de Madrid (1995).

[39] J.F. Padial, J.M.Rakotoson and L. Tello, Introduction to the monotone and relative rearrangements and applications, Rapport, Département de Mathématiques, Université de Poitiers (1993).

[40] G. Pòlya and W.N. Szegö, Isopermetric inequalities in mathematical physics, Princenton Univ. Press (1951).

[41] J.P. Puel, A nonlinear eigenvalue problem with free boundary. C.R. Acad. Sci. Paris A 284 (1977) 861-863.

[42] J.M. Rakotoson, Some properties of the relative rearrangement. J. Math. Anal. Appl. 135 (1988) 488-500.

[43] J.M. Rakotoson, A differentiability result for the relative rearrangement. Diff. Int. Eq. 2 (1989) 363-377.

[44] J.M. Rakotoson, Relative rearrangement for highly nonlinear equations. Nonlinear Analysis. Theory, Meth. and Appl. 24 (1995) 493-507.

[45] J.M. Rakotoson and M.L. Seoane (in preparation).

[46] J.M. Rakotoson, Galerkin approximations, strong continuity of the relative rearrangement map and application to plasma physics equations. Diff. Int. Eq. 12 (1999) 67-81.

[47] J.M. Rakotoson and B. Simon, Relative rearrangement on a measure space. Application to the regularity of weighted monotone rearrangement. Part I-II. Appl. Math. Lett. 6 (1993) 75-78; 79-92.

[48] J.M. Rakotoson and B. Simon, Relative rearrangement on a finite measure space. Application to weighted spaces and to P.D.E. Rev. R. Acad. Cienc. Exactas Fís. Nat. (Esp.) 91 (1997) 33-45.

[49] J.M. Rakotoson and R. Temam, A co-area formula with applications to monotone rearrangement and to regularity. Arch. Rational Mech. Anal. 109 (1991) 213-238.

[50] R.T. Rockafellar, Convex Analysis, Princeton University Press (1970).

[51] V.D. Shafranov, On agneto-hydrodynamical equilibriium configurations. Soviet Physics JETP, 6 (1958) 5456-554.

[52] G.G. Talenti, Rearrangements of functions and and Partial Differential Equations. Nonlinear Diffusion Problems, A. Fasano and M. Primicerio Eds, Springer-Verlag (1986) 153-178.

[53] G.G. Talenti, Rearrangements and PDE. Inequalities, fifty years on from Hardy, Littlewood and Pòlya, W.N. Everitt Ed., Marcel Dekker Inc (1991) 211-230.

[54] G.G. Talenti, Assembling a rearrangement. Arch. Rat. Mech. Anal. 98 (1987) 85-93

[55] R.Temam, A nonlinear eigenvalue problem: equilibrium shape of a confined plasma. Arch. Rat. Mech. Anal. 65 (1975) 51-73.

[56] R.Temam, Remarks on a free boundary problem arising in plasma physics. Comm. Par. Diff. Eq. 2 (1977) 563-585.

[57] R.Temam, Monotone rearrangement of functions and the Grad-Mercier equation of plasma physics, Proc. Int. Conf. Recent Methods in Nonlinear Analysis and Applications, E. de Giogi and U. Mosco Eds (1978).

[58] R.Temam, Analyse Numerique, Presses Universitaires de France (1971).

[59] J.F. Toland, Duality in nonconvex optimization. J. Math. Appl. 66 (1978) 399-415.

[60] J.F. Toland, A Duality Principle for Non-convex Optimisation and the Calculus the Variations. Arch. Rat. Mech. Anal. 71 (1979) 41-61.

[61] R.S. Varga, Matrix Iterative Analysis, Prentice-Hall Inc. (1962) 